\title{
Deubiquitylating enzymes and drug discovery: emerging opportunities
}

\author{
Jeanine A. Harrigan ${ }^{*}$, Xavier Jacq ${ }^{*}$, Niall M. Martin ${ }^{1,2}$, and Stephen P. Jackson ${ }^{1,3}$ \\ Abstract | More than a decade after a Nobel Prize was awarded for the discovery of the ubiquitin- \\ proteasome system and clinical approval of proteasome and ubiquitin E3 ligase inhibitors, \\ first-generation deubiquitylating enzyme (DUB) inhibitors are now approaching clinical trials. \\ However, although our knowledge of the physiological and pathophysiological roles of DUBs has \\ evolved tremendously, the clinical development of selective DUB inhibitors has been challenging. \\ In this Review, we discuss these issues and highlight recent advances in our understanding of \\ DUB enzymology and biology as well as technological improvements that have contributed to \\ the current interest in DUBs as therapeutic targets in diseases ranging from oncology \\ to neurodegeneration.
}

\section{Ubiquitin}

A small protein that is

conjugated to other proteins

(including itself) as a

post-translational modification often to control cellular

signalling or degradation of the modified protein.

Ubiquitin-like proteins (UBLs). Proteins such as small ubiquitin-like modifier (SUMO) or interferon-stimulated gene 15 (ISG15) that adopt a $\beta$-grasp fold, which is characteristic of ubiquitin and related proteins.

'Mission Therapeutics Ltd, Moneta, Babraham Research Campus, Cambridge CB22 3AT, UK

2Present address: Artios Pharmaceuticals Ltd, Maia, Babraham Research Campus, Cambridge CB22 3AT, UK

${ }^{3}$ The Wellcome Trust and Cancer Research UK Gurdon Institute, and Department of Biochemistry, Tennis Court Road, University of Cambridge, Cambridge CB2 1ON, UK

*These authors contributed equally to this work.

Correspondence to S.P.J. s.jackson@gurdon.cam.ac.uk
The sequential enzymatic processes that covalently attach ubiquitin, a 76-residue polypeptide, to target proteins - a process known as ubiquitylation - are now well understood ${ }^{1}$ (FIG. 1a). In some cases, a single ubiquitin is attached to the target protein, whereas in others, multiple monoubiquitin adducts are conjugated to different residues of the target. In many instances, various types of ubiquitin chains are produced, wherein one ubiquitin moiety is attached to a free amino group of another. This leads to linear ubiquitin chains and chains involving internal ubiquitin lysine residues K6, $\mathrm{K} 11, \mathrm{~K} 27, \mathrm{~K} 29$, K33, K48, K63, as well as mixed ubiquitin chains containing different linkages, or linkages between ubiquitin and ubiquitin-like proteins (UBLs) that include small ubiquitin-like modifier (SUMO) and neuronal precursor cell-expressed developmentally downregulated protein 8 (NEDD8).

These different types of ubiquitin and UBL modifications, sometimes referred to as 'the ubiquitin code', have specific and diverse effects on protein and cell physiology. For example, such modifications can target proteins that are damaged or improperly folded, or that have intrinsically short half-lives for degradation via the ubiquitin-proteasome system (UPS) ${ }^{2}$. Appropriately polyubiquitylated proteins are recognized and degraded by the $26 \mathrm{~S}$ macromolecular proteasome complex ${ }^{3}$ via mechanisms that have been extensively reviewed elsewhere ${ }^{4,5}$. In other instances, ubiquitylation regulates protein interactions, localization and enzymatic activities, thereby affecting cellular processes, including transcription, DNA damage signalling and DNA repair, cell cycle progression, endocytosis, apoptosis and various other processes ${ }^{6-9}$. Such control mechanisms often involve ubiquitin-binding proteins, many of which exist in eukaryotic cells ${ }^{10}$. The recent demonstration of post-translational modification of ubiquitin itself provides an additional layer of regulation that affects various cellular processes ${ }^{11}$.

Like other post-translational modifications, ubiquitylation is reversible: peptidases termed deubiquitylating enzymes (DUBs) can cleave ubiquitin from substrate proteins, edit ubiquitin chains and process ubiquitin precursors $^{12}$. Some DUBs and related enzymes are involved in editing or processing UBLs and their conjugates ${ }^{13}$; prime examples of these being the SENP (sentrin/SUMOspecific protease) proteins that process SUMO precursors and SUMO conjugates ${ }^{14}$. DUBs are classified into six families based on sequence and domain conservation (FIG. 1 b): USPs (ubiquitin-specific proteases), UCHs (ubiquitin carboxy-terminal hydrolases), MJDs (Machado-Josephin domain-containing proteases), OTUs (ovarian tumour proteases), MINDYs (motif-interacting with ubiquitincontaining novel DUB family) and JAMMs (JAB1, MPN, MOV34 family). SENPs and the first five DUB families are cysteine peptidases, whereas JAMMs are zinc metallopeptidases.

Ubiquitylation and related processes control myriad aspects of human cell biology and physiology, and defects in such processes contribute to many diseases. Accordingly, DUB deregulation contributes to various sporadic and genetic disorders. Notable examples include: the UCH family member BRCA1-associated protein 1 (BAP1), mutated in melanoma, mesothelioma and renal cell carcinoma ${ }^{15}$; USP6, translocated in aneurysmal bone cysts ${ }^{16}$; USP7, mutated in neurological disorders ${ }^{17}$; USP8, whose mutations cause Cushing disease $^{18,19}$; USP9X, whose mutations cause developmental disorders ${ }^{20}$ and whose expression is dysregulated 
a
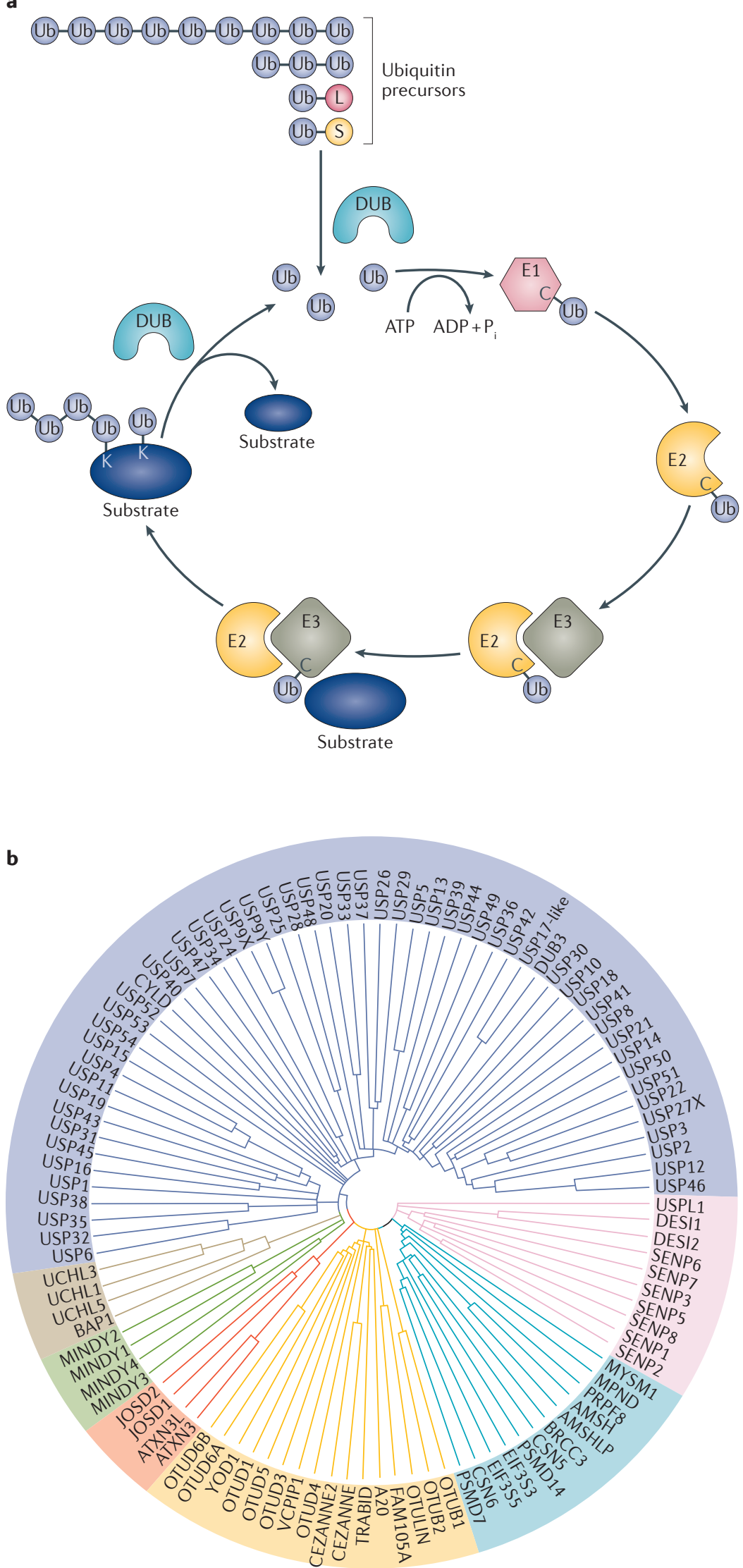

in cancer ${ }^{21}$; USP15, amplified in certain glioblastoma, breast and ovarian cancers ${ }^{22}$; and CYLD, commonly mutated in cylindromatosis ${ }^{23}$. Deregulation of MJDfamily DUBs has also been linked to diseases associated with polyglutamine amplification. For example, expansion of DNA 'CAG' trinucleotide repeats in ataxin 3 (ATXN3) causes Machado-Joseph disease (also known as spinocerebellar ataxia 3$)^{24}$. Furthermore, mutations in the JAMM family member associated molecule with the SH3 domain of STAM (AMSH; also known as STAMBP) cause microcephaly-capillary malformation syndrome ${ }^{25}$.

There has been growing interest in exploiting components of the ubiquitylation machinery as therapeutic targets ${ }^{26}$. Although there has been strong progress in developing small-molecule inhibitors of ubiquitin and UBL E1 enzymes ${ }^{27}$, the highly pleiotropic nature of E1s means that such drugs will likely be confined to acute settings, such as in the treatment of aggressive cancers. Given their greater numbers and diversity, E2s, E3s and DUBs offer the potential for developing drugs with more specific effects. In particular, being a group of diverse enzymes with well-defined catalytic clefts, DUBs are intrinsically attractive as potential drug targets ${ }^{26}$. However - as we discuss further below - until recently, the development of selective DUB inhibitors has been

Figure 1 The ubiquitylation cascade and the deubiquitylase family of proteins. a|Schematic of key events in ubiquitylation and deubiquitylation. The E1 enzyme activates ubiquitin in an ATP-dependent manner, resulting in a covalent thioester linkage between ubiquitin and the $\mathrm{E} 1$ cysteine residue. Ubiquitin is then transferred to an E2 conjugating enzyme, forming a thioester linkage with the catalytic cysteine. Finally, an E3 ligase assists or directly catalyses the transfer of ubiquitin from the E2 to a substrate, usually via a lysine side chain. An example of a HECT (homologous to the E6AP carboxyl terminus) or RBR (RING-between-RING) E3 ligase is shown. In subsequent rounds, ubiquitin molecules can be conjugated to the $\mathrm{N}$-terminal amino group or lysines on ubiquitin itself to form chains. Deubiquitylating enzymes (DUBs) remove ubiquitin molecules from substrates or process ubiquitin precursors to generate free ubiquitin pools. $\mathbf{b} \mid$ DUB phylogenetic tree. Sequences for full-length DUB and SENP (sentrin/SUMO-specific protease) proteins were aligned with COBALT (constraint-based multiple alignment tool), a computational tool for multiple protein sequences, and subsequently visualized with FigTree v1.4.3. In regard to USP17-like, note that various related human USP17-like DUBs exist. AMSH, associated molecule with the $\mathrm{SH} 3$ domain of STAM; AMSHLP, AMSH-like protease; ATXN3, ataxin 3; BAP1, BRCA1-associated protein 1; CEZANNE, cellular zinc finger anti-NF- $\kappa B$ protein; $\mathrm{CSN}$, COP9 signalosome complex subunit; CYLD, cylindromatosis; DESI, desumoylating isopeptidase; EIF3, eukaryotic translation initiation factor 3; JAMM, JAB1, MPN, MOV34 family; JOSD, josephin domain; MINDY, motif-interacting with ubiquitin-containing novel DUB family; MJD, Machado-Josephin domain-containing protease; OTUD, OTU domain-containing protein; PRPF8, pre-mRNA-processing splicing factor 8; UCHL, ubiquitin carboxy-terminal hydrolase-like; USP, ubiquitin-specific protease; VCPIP1, valosin-containing protein p97/p47 complex-interacting protein 1. 
limited by insufficient understanding of DUB biology, difficulties in establishing robust biochemical assays suitable for compound screening, limitations in cellular and in vivo models to assess DUB activity or inhibition, and the pleiotropic nature of various small-molecule DUB inhibitors. With many of these issues now being largely overcome, the rate of progress in DUB drug discovery has accelerated over the past few years, with various selective compounds being described and characterized by both academic groups and companies.

In this Review, we discuss how DUBs and their deregulation affect human diseases, particularly cancer, neurodegeneration and inflammation (TABLE 1), and highlight the therapeutic potential for pharmacological modulation of DUB activities. Recent advances in assay development and screening technologies, which are enabling researchers and drug developers to overcome recurrent challenges in the clinical translation of DUB inhibitors, are also discussed.

\section{DUBs in oncology}

Accumulating evidence implicates DUBs in tumorigenesis at multiple levels (FIG. 2). First, DUBs such as BAP1, UCHL1 and CYLD have been described as displaying intrinsic oncogenic or tumour suppressor activities $^{28}$. Second, some DUBs, such as USP22, are connected to controlling key epigenetic changes that promote tumour development ${ }^{29}$. Third, through their deubiquitylating activities, various DUBs, such as USP7 and USP28, have been reported to regulate the levels and/or activities of various oncogene or tumour suppressor proteins ${ }^{30,31}$. Fourth, DUBs modulate other therapeutically relevant cellular components and processes, such as the UPS (for example, USP14 and UCHL5 (also known as $\mathrm{UCH} 37)$ ) $^{32}$, stem cell renewal (for example, USP16 or USP22) 29,33 , DNA damage response (DDR) and DNA repair (for example, USP1 or USP11) ${ }^{9}$, immunooncology (for example, USP7) ${ }^{34}$ or receptor tyrosine kinases (for example, USP8 or USP9X) ${ }^{35,36}$. Consequently, and as described in more detail below, various DUBs are emerging as attractive targets for the development of novel cancer therapies.

\section{Proteasomal DUBs}

The successful targeting of the proteasome for cancer therapy is underlined by the clinical success of bortezomib, a broadly acting proteasome inhibitor, in refractory multiple myeloma ${ }^{37}$ or mantle cell myeloma ${ }^{38}$. However, three DUBs associated with proteasome functions - PSMD14 (also known as POH1), USP14 and UCHL5 - may represent more specific anticancer targets. To facilitate the degradation of proteasome-targeted substrates, these specialized DUBs remove ubiquitin moieties that would otherwise impede entry into the 20 S proteasome catalytic core ${ }^{39}$.

The JAMM metalloprotease PSMD14 has been highlighted as a potential therapeutic target through studies showing that its levels inversely correlate with survival of patients with multiple myeloma and that its depletion impairs proliferation of multiple myeloma cells ${ }^{40}$. In addition, nuclear PSMD14 is elevated in hepatocellular carcinomas and correlates with E2F1 overexpression and tumour growth ${ }^{41}$. PSMD14 has also been reported to regulate the ubiquitylation and stability of the oncogene receptor tyrosine kinase ERBB2 (REF. 42). Furthermore, as PSMD14 has been connected to promoting cellular responses to DNA double-strand breaks, particularly by the process of homologous recombination, PSMD14 inhibition could potentially sensitize cancer cells to DNA-damaging agents and/or preferentially kill cancer cells that rely strongly on homologous recombination ${ }^{43}$.

Another potential anticancer therapeutic target is USP14, which is primarily associated with the proteasome 19S regulatory subunit, where it potentiates ubiquitin recycling ${ }^{44}$. USP14 is not constitutively active but reversibly associates with the 19S RPN1 subunit (also known as PSMD2), which enhances its activity ${ }^{45}$. USP14 inhibits proteasomal degradation of ubiquitin-protein conjugates by trimming ubiquitin chains on protein substrates before their degradation ${ }^{46}$. USP14 expression is upregulated in non-small-cell lung cancer, especially in adenocarcinoma ${ }^{47}$, and its levels are reportedly elevated in ovarian cancer samples ${ }^{48}$. In line with this, USP14 is connected to several important signalling pathways, for example, as a substrate of AKT mediating intracellular signalling for growth factors ${ }^{49}$ and a modulator of Dishevelled proteins, key positive regulators of WNT signalling ${ }^{50}$.

Like USP14, the DUB UCHL5 reversibly interacts with the proteasome $\mathrm{e}^{51}$, binding to the RPN13 receptor (also known as ADRM1) ${ }^{52}$ in a manner that enhances UCHL5 isopeptidase activity ${ }^{51,53}$. A key function of UCHL5 is to remove distal ubiquitin moieties from polyubiquitylated proteins, thereby liberating proteins from destruction ${ }^{54}$, or facilitating destruction of certain substrates, as described for inducible nitric oxide synthase and nuclear factor- $\kappa \mathrm{B}$ inhibitor- $\alpha(\mathrm{I} \kappa \mathrm{B} \alpha)^{55}$. It therefore seems that, like USP14, UCHL5 suppresses the destruction of certain proteins, while promoting the degradation of others. Notably, RNA interference studies showed that depletion of either USP14 or UCHL5 alone had no detectable effect on cell growth, proteasome structure or proteolytic capacity but did accelerate cellular protein degradation ${ }^{53}$. By contrast, depletion of both DUBs decreased protein degradation, suggesting that they have overlapping functions. UCHL5 is overexpressed in epithelial ovarian cancer, which is associated with advanced tumour progression and poor clinical outcome ${ }^{56}$. UCHL5 is also overexpressed in hepatocellular carcinoma, and was shown to promote cell migration and invasion ${ }^{57}$.

These proteasome-associated DUBs represent attractive drug targets, as their inhibition might have substantial effects on cancer cell physiology but with fewer toxicities than are seen with drugs targeting core proteasome catalytic function ${ }^{58}$. Indeed, VLX1570 (TABLE 2), the most advanced reported DUB inhibitor, which was recently in phase I trials (now suspended) for treatment of multiple myeloma and solid tumours ${ }^{59}$, has been described to target USP14 and UCHL5 (REF. 60). VLX1570 is a ring-expanded version of the compound b-AP15 (also known as VLX1500) that was identified 
Table 1 | DUBs connected with human diseases

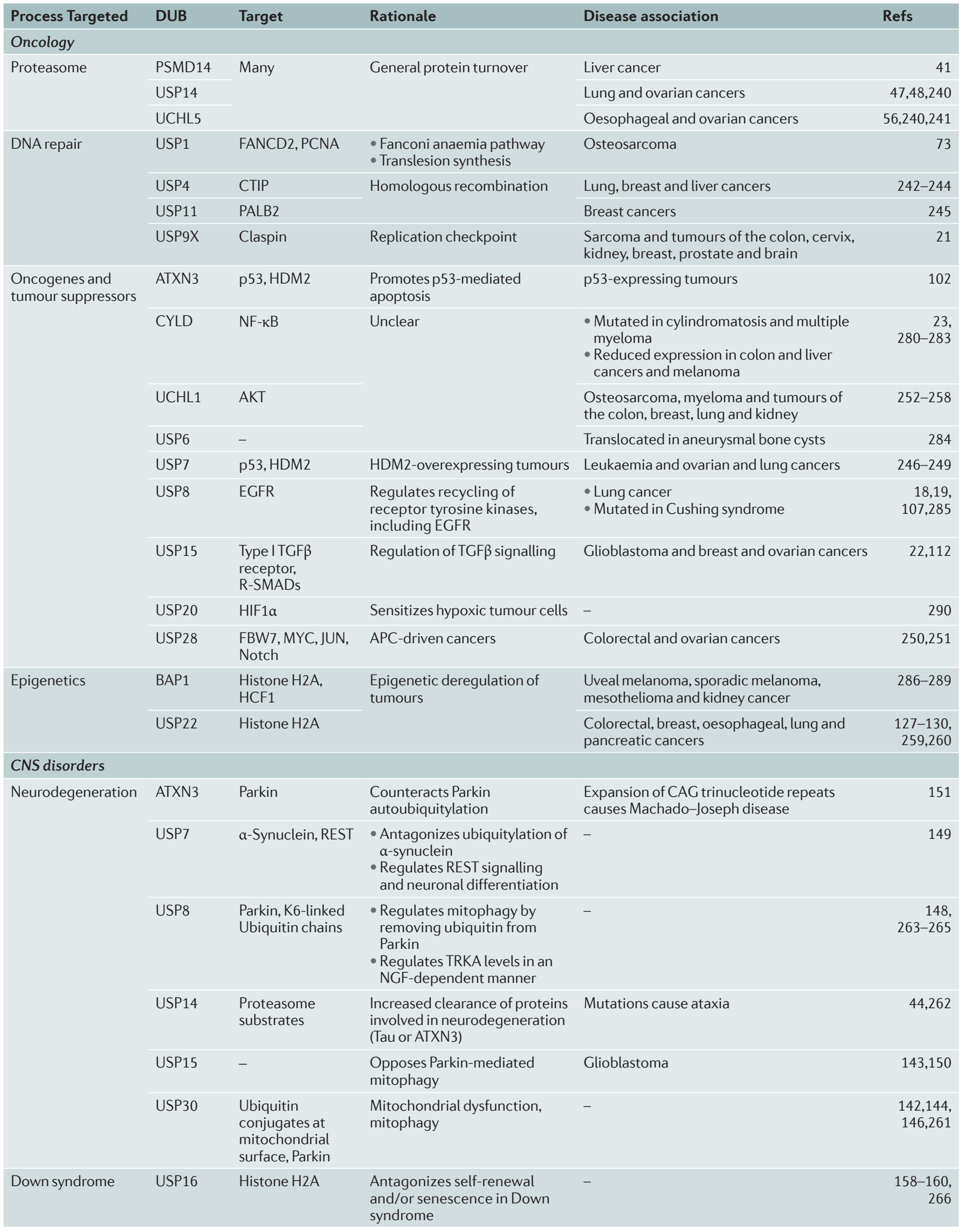


Table 1 (cont.) | DUBs connected with human diseases

\begin{tabular}{|c|c|c|c|c|c|}
\hline Process Targeted & DUB & Target & Rationale & Disease association & Refs \\
\hline \multicolumn{6}{|c|}{ Inflammation, immunity and infectious disease } \\
\hline \multirow{4}{*}{$\begin{array}{l}\text { Negative regulation } \\
\text { of the immune } \\
\text { response }\end{array}$} & A20 & $\begin{array}{l}\text { NEMO, RIPK1, } \\
\text { TRAF6 }\end{array}$ & \multirow[t]{3}{*}{ Inhibits NF- $\kappa B$ signalling } & $\begin{array}{l}\text { Expression levels regulated by TNF } \alpha, \text { IL-1 } \beta \\
\text { and LPS }\end{array}$ & $\begin{array}{r}167, \\
273,274\end{array}$ \\
\hline & CYLD & RIG1, TBK1, IKK $\varepsilon$ & & - & 170,171 \\
\hline & OTULIN & $\begin{array}{l}\text { RIPK1, RIPK2, } \\
\text { NEMO }\end{array}$ & & - & 172,173 \\
\hline & USP25 & $\begin{array}{l}\text { RIG1, TRAF2, } \\
\text { TRAF3, TRAF6 }\end{array}$ & $\begin{array}{l}\text { - Negatively regulates } \\
\text { IL-17-triggered signalling } \\
\text { - Negatively regulates } \\
\text { virus-induced type I IFN } \\
\text { production } \\
\text { - Positive feedback regulation } \\
\text { of innate immune } \\
\text { responses against RNA and } \\
\text { DNA viruses }\end{array}$ & Expression regulated by IFN and IRF7 & $\begin{array}{r}270-272, \\
292,303\end{array}$ \\
\hline \multirow[t]{2}{*}{$\mathrm{T}_{\text {reg }}$ responses } & USP7 & FOXP3 & $\begin{array}{l}\text { - Stabilizes FOXP3 in } \\
\mathrm{T}_{\text {reg }} \text { cells } \\
\text { - Negative regulator of } \\
\text { TNF } \alpha \text {-stimulated NF- } \mathrm{kB} \\
\text { activity }\end{array}$ & $\begin{array}{l}\text { Expressed and regulated upon viral } \\
\text { infections in B and T cells }\end{array}$ & $34,267,293$ \\
\hline & USP21 & FOXP3 & Stabilizes FOXP3 in $\mathrm{T}_{\text {reg }}$ cells & - & 131 \\
\hline \multirow[t]{4}{*}{$\begin{array}{l}\mathrm{T}_{\mathrm{H}} 1 \text { and } \mathrm{T}_{\mathrm{H}} 17 \\
\text { responses }\end{array}$} & CEZANNE & ZAP70 & $\begin{array}{l}\text { - Positive regulator of T cell } \\
\text { receptor signalling } \\
\text { - Binds to and deubiquitylates } \\
\text { ZAP70 }\end{array}$ & - & 193 \\
\hline & USP10 & T-bet & Stabilizes T-bet in $T_{H} 1$ cells & $\begin{array}{l}\text { Highly expressed in PBMCs from patients } \\
\text { with asthma }\end{array}$ & 196 \\
\hline & USP17 & $\begin{array}{l}\text { ROR } \gamma t, \text { RIG1, } \\
\text { IL-33 }\end{array}$ & $\begin{array}{l}\text { - Positive regulator of ROR } \gamma \mathrm{t} \text { in } \\
\mathrm{T}_{\mathrm{H}} 17 \text { cells } \\
\text { - Regulates virus-induced } \\
\text { type I IFN signalling } \\
\text { - Regulates the stability and } \\
\text { nuclear function of IL-33 }\end{array}$ & - & $\begin{array}{r}277-279 \\
295\end{array}$ \\
\hline & USP18 & $\begin{array}{l}\text { TAK1-TAB1 } \\
\text { complex }\end{array}$ & $\begin{array}{l}\text { Regulates TAK1-TAB1 } \\
\text { interaction required for } \mathrm{T}_{\mathrm{H}} 17 \\
\text { differentiation }\end{array}$ & Expression induced by cytokines & 192 \\
\hline
\end{tabular}

APC, adenomatous polyposis coli protein; ATXN3, ataxin 3; BAP1, BRCA1-associated protein 1; CEZANNE, cellular zinc finger anti-NF- $\mathrm{KB}$ protein; CNS, central nervous system; CYLD, cylindromatosis; CTIP, C-terminal-binding protein-interacting protein; EGFR, epidermal growth factor receptor; FANCD2, Fanconi anaemia group D2 protein; FBW7, F-box and WD40 domain-containing protein 7; FOXP3, forkhead box protein P3; HCF1, host cell factor 1; HDM2, human double minute 2;

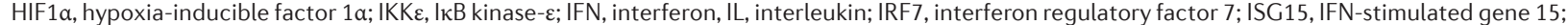
JMJD2D, Jumonji domain-containing protein 2D; LPS, lipopolysaccharide; NEMO, NF-kB essential modulator; NF-kB, nuclear factor-kB; NGF, $\beta$-nerve growth factor; PALB2, partner and localizer of BRCA2; PBMC, peripheral blood mononuclear cell; PCNA, proliferating cell nuclear antigen; REST, RE1-silencing transcription factor; RIG1, retinoic acid-inducible gene 1-like receptor 1; RIPK, receptor-interacting serine/threonine-protein kinase; ROR $\gamma t$, retinoid-related orphan receptor- $\gamma t$ t; R-SMAD, receptor-regulated SMAD; TAB, TAK1-binding protein; TAK1, TGF $\beta$-activated kinase 1; TBK1, TANK-binding kinase 1; TGF $\beta$, transforming growth factor- $\beta ; \mathrm{T}_{H}$ cells, T helper cells; TLR, Toll-like receptor; TNF $\alpha$, tumour necrosis factor- $\alpha$; TRAF6, TNF receptor-associated factor $6 ; T_{\text {rec }}$ cells, regulatory T cells; TRKA, tropomyosin-related kinase A; UCHL, ubiquitin carboxy-terminal hydrolase-like; USP, ubiquitin-specific protease; ZAP70, 70 kDa $\zeta$-chain-associated protein. 


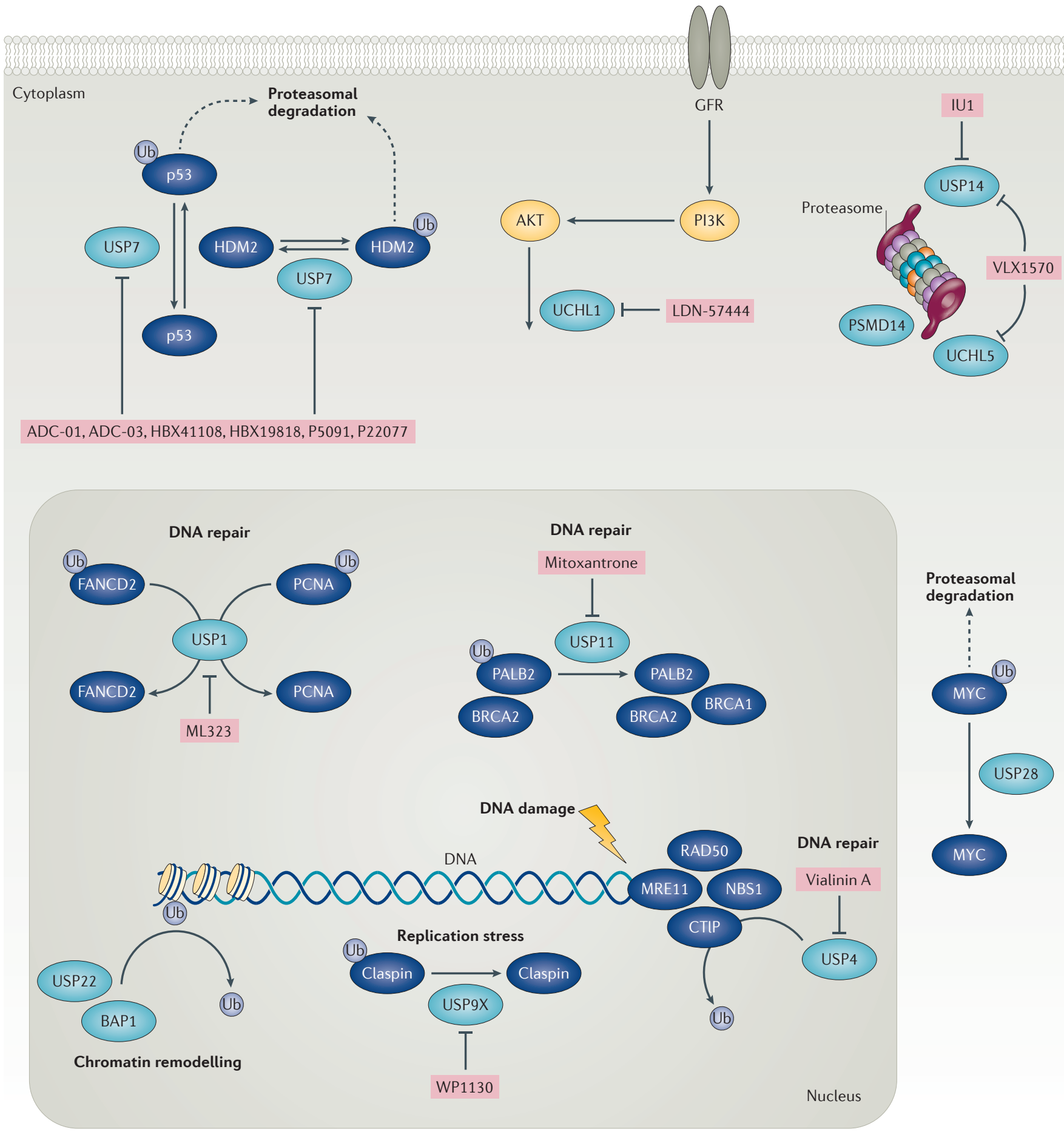

Figure 2 | Various roles of DUBs in oncology. Selected, representative examples of deubiquitylating enzymes (DUBs; light blue ovals) involved in distinct cellular pathways and regulation of various ubiquitylated substrates (dark blue ovals) related to oncology. The proteasome and associated DUBs facilitate protein turnover and recycle ubiquitin. Ubiquitin-specific protease 28 (USP28) regulates turnover of the oncogene product MYC, ataxin 3 (ATXN3) controls the stability of the tumour suppressor $\mathrm{p} 53$, and USP7 regulates $\mathrm{p} 53$ and its $\mathrm{E} 3$ ubiquitin ligase human double minute 2 (HDM2). USP1, USP4 and USP11 have important roles in DNA damage repair, whereas USP9X regulates claspin and is linked to replication stress and checkpoint signalling. BRCA1-associated protein 1 (BAP1) and USP22 participate in chromatin remodelling by deubiquitylating histones, and ubiquitin carboxy-terminal hydrolase-like 1 (UCHL1) plays a part in AKT signalling. These are representative examples only and not meant to be exhaustive. Examples of small-molecule compounds targeting these DUBs are shown. BRCA1, breast cancer type 1 susceptibility protein; CTIP, C-terminal-binding protein-interacting protein; FANCD2, Fanconi anaemia group D2 protein; GFR, growth factor receptor; MRE11, meiotic recombination 11 homologue 1; NBS1, Nijmegen breakage syndrome protein 1; PALB2, partner and localizer of BRCA2; PCNA, proliferating cell nuclear antigen; PI3K, phosphoinositide 3-kinase. 
from cell-based screens looking for compounds inducing p53-independent apoptosis. Cells treated with b-AP15 accumulate polyubiquitin chains ${ }^{61}$, and it has been claimed that b-AP15 targets USP14 and possibly also UCHL5 (REF. 60). This compound was reported to be reversible and reasonably selective against other $\mathrm{DUBs}^{60}$ in a cell-based activity probe assay, with a median inhibitory concentration $\left(\mathrm{IC}_{50}\right.$ ) of $\sim 2 \mu \mathrm{M}$ against purified 19S proteasome DUB activities. b-AP15 displayed strong activity when tested in various in vivo solid tumour model ${ }^{59}$, including multiple myeloma ${ }^{62}$, but it remains to be seen whether VLX1570 selectivity will be sufficient to deliver on its promise as a nextgeneration proteasome inhibitor. Cleave Biosciences has also published a series of patent applications describing compounds that inhibit JAMM proteases, providing potential angles for developing selective PSMD14 inhibitors $^{63-65}$ (TABLE 2).

\section{DUBs linked to DNA repair}

One hallmark of cancer is the downregulation, loss or deregulation of certain DNA repair and DDR pathways and/or strong reliance on such pathways ${ }^{66,67}$. DNA repair and DDR mechanisms are regulated by posttranslational modifications, such as ubiquitylation, and many DUBs are strongly linked to such processes ${ }^{9,68}$.

One example of this is USP1, a DUB identified as a regulator of Fanconi anaemia group D2 protein (FANCD2) ubiquitylation, a key protein involved in the Fanconi anaemia pathway of DNA crosslink repair ${ }^{69,70}$. USP1 influences accumulation of the Fanconi anaemia core complex at DNA damage sites and deubiquitylates FANCD2-FANCI in a cell cycle-dependent manner ${ }^{69}$. USP1 also removes monoubiquitin from proliferating cell nuclear antigen (PCNA), a DNA replication component that also functions in DNA repair by translation synthesis $^{71}$. Other USP1 activities include involvement in a feedback loop to limit DDR CHK1 protein kinase activity $^{72}$, and the regulation of cellular differentiation in osteosarcoma cells by deubiquitylating and hence affecting the stability of inhibitors of DNA-binding proteins ${ }^{73}$. In vitro, USP1 activity is greatly stimulated by USP1-associated factor 1 (UAF1; also known as WDR48), enhancing USP1 catalytic turnover $\left(k_{\text {cat }}\right)$ but not affinity $\left(K_{\mathrm{m}}\right)$ for monoubiquitylated substrates ${ }^{74}$. Selective USP1 inhibitors with submicromolar potency have been identified ${ }^{75}$, and one of these, pimozide, was shown to re-sensitize platinum-resistant non-small-cell lung cancer cells and promote FANCD2 and PCNA monoubiquitylation ${ }^{75}$. However, although these studies indicated on-target effects, DUB selectivity profiling suggested that pimozide might be less selective than initially described ${ }^{76}$. Optimization of certain USP1 screening hits has generated additional molecules ${ }^{77}$, most notably a selective pyrimidine core compound, ML323 (TABLE 2). This molecule allosterically blocks complex formation between UAF1 and USP1 (REF. 78), potentiates cisplatin cytotoxicity, and increases PCNA and FANCD2 monoubiquitylation in cells $\mathrm{s}^{77}$. So far, however, little progress has been made in advancing selective USP1 inhibitors into clinical development.
Another DUB linked to DNA repair is USP11, which was initially described to form a complex with the DDR tumour suppressor breast cancer type 2 susceptibility protein (BRCA2) to promote the DNA double-strand break repair pathway of homologous recombination ${ }^{79}$. Depletion of USP11 has been shown to sensitize cells to olaparib (also known as AZD2281), which inhibits the DDR enzyme poly(ADP-ribose) polymerase 1 (PARP 1$)^{80}$. Recently, an interaction between BRCA1 and partner and localizer of BRCA2 (PALB2) - which functionally cooperates with BRCA2 in DNA repair - was shown to be under ubiquitin control, with PALB2 ubiquitylation suppressing its interaction with BRCA1 in a manner counteracted by USP11 (REF. 81).

The only currently reported USP11 inhibitor is the topoisomerase inhibitor mitoxantrone ${ }^{82}$ (TABLE 2). Although the authors reported low nanomolar potency in a pancreatic ductal adenocarcinoma cell survival model, no further development of this compound has been reported. Given the apparent amenability of USP11 to small-molecule inhibition, it is notable that USP4, a DUB closely related to USP11, was recently shown to be involved in the DDR through promoting early stages of homologous recombination ${ }^{83}$.

$\mathrm{USP}^{21}{ }^{21}$, which maintains DNA replication fork stability and DNA damage checkpoint responses by regulating the protein claspin during $S$ phase $\mathrm{e}^{84}$, may represent another potential therapeutic target. USP9X has been shown to affect radiosensitivity in glioblastoma cells by myeloid cell leukaemia 1 (MCL1)-dependent and -independent mechanisms ${ }^{85}$. The best-described USP9X inhibitor is WP1130 (TABLE 2), identified in a screen for Janus kinase 2 (JAK2) inhibitors, which was shown to inhibit USP9X as well as other DUBs (USP5, USP14 and UCHL5 $)^{86,87}$. It was shown by mass spectrometry that the covalent mechanism of action of this compound is reversible ${ }^{76}$.

\section{Regulation of oncogenes and tumour suppressors}

Various DUBs have been reported to have connections to tumour-suppressing or oncogenic functions, and may therefore represent potential therapeutic targets ${ }^{88}$.

p53 regulation. Several DUBs have been linked to regulation of the tumour suppressor protein p53, which has pivotal roles in cellular stress responses and is lost or mutated in many cancers ${ }^{89}$. Human double minute 2 (HDM2; also known as MDM2) is a RING-type ubiquitin E3 ligase and key negative regulator of p53 through its ability to ubiquitylate p53 and target it for degradation $^{90}$. By cleaving ubiquitin chains on HDM2, USP7 counteracts HDM2 proteasomal degradation, leading to 553 suppression through increased ubiquitylation and degradation ${ }^{91,92}$. In theory, USP7 inhibition should therefore trigger HDM2 degradation, p53 stabilization and ultimately activation of apoptotic pathways in tumour cells ${ }^{93}$. Additional USP7 targets have also been described, such as phosphatase and tensin homologue (PTEN), forkhead box protein O4 (FOXO4) and FOXP3 (REFS 34,94,95), suggesting alternative 


\section{REVIEWS}

Table 2 | DUB inhibitors in development

\begin{tabular}{|c|c|c|c|c|c|c|}
\hline DUB & Inhibitor & Structure* & $\begin{array}{l}\text { Company/ } \\
\text { Institution }\end{array}$ & $\begin{array}{l}\text { Disease } \\
\text { indication }\end{array}$ & $\begin{array}{l}\text { Stage of } \\
\text { development }\end{array}$ & Refs \\
\hline UCHL1 & LDN-57444 & & $\begin{array}{l}\text { Brigham and } \\
\text { Women's Hospital } \\
\text { and Harvard } \\
\text { Medical School }\end{array}$ & Oncology & Preclinical & 120 \\
\hline $\begin{array}{l}\text { UCHL5 } \\
\text { and } \\
\text { USP14 }\end{array}$ & VLX1570 & & Vivolux & Oncology & $\begin{array}{l}\text { Clinical trial } \\
\text { phase (now } \\
\text { suspended) }\end{array}$ & 296 \\
\hline USP2 & ML364 & & $\begin{array}{l}\text { National Institutes } \\
\text { of Health }\end{array}$ & Inflammation & Preclinical & 301 \\
\hline USP4 & Vialinin A & & $\begin{array}{l}\text { Tokyo University } \\
\text { of Agriculture and } \\
\text { Shanghai Institutes } \\
\text { for Biological } \\
\text { Sciences }\end{array}$ & $\begin{array}{l}\text { Inflammation and } \\
\text { oncology }\end{array}$ & Preclinical & 189,190 \\
\hline
\end{tabular}


Table 2 (cont.) | DUB inhibitors in development

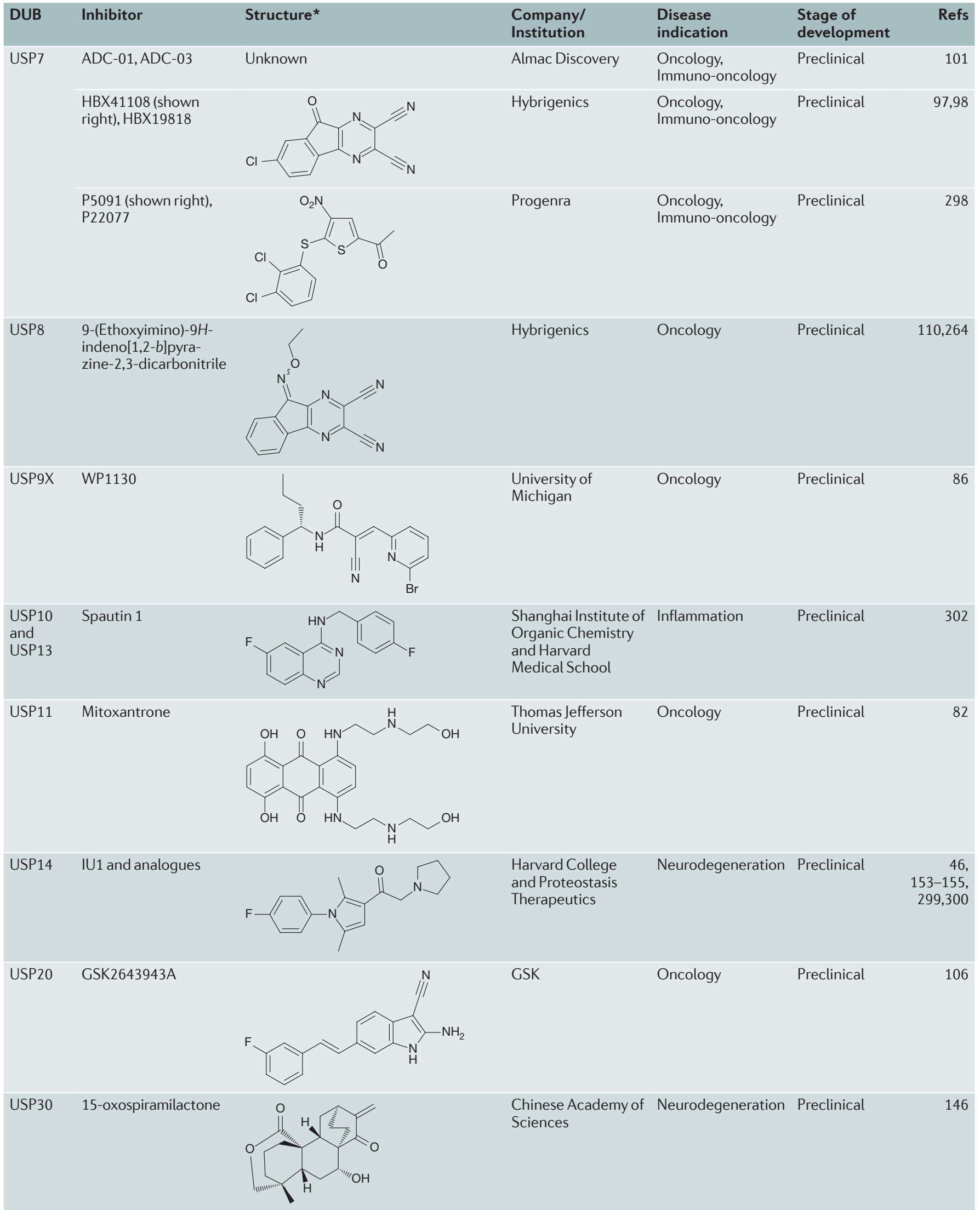

*Chemical structures shown are representative only, and additional inhibitors and an assessment of their drug-likeness and reproducibility can be found in Kemp, 2016 (REF. 210). GSK, GlaxoSmithKline; UCHL, ubiquitin carboxy-terminal hydrolase-like; USP, ubiquitin-specific protease. 
therapeutic mechanisms for USP7 inhibitors. USP7 has also recently been shown to promote DNA replication via acting as a DUB for the UBL SUMO ${ }^{96}$.

The first published submicromolar USP7 inhibitor HBX41108 (REF. 97) was shown to be a rather nonspecific inhibitor of DUBs ${ }^{76}$. Recently, more-selective amidotetrahydroacridine derivatives such as HBX19818 and HBX28258 were identified, although these exhibited fairly low potency ${ }^{98}$. Despite this, HBX19818 was shown to covalently bind to the catalytic Cys residue of USP7 in preference to other cysteinyl groups, and to stabilize p53 and promote G1 arrest and apoptosis in cells $^{98}$. Progenra's thiophene chemical series also provided relatively nonspecific USP7 inhibitors, including the compounds P5091 and P22077 (REF. 99). In multiple myeloma cells, P5091 stabilized p53 and inhibited tumour growth, whereas in animal models, P5091 was well tolerated, inhibited tumour growth and prolonged survival $^{99}$. More recent in vivo studies using P22077 within an orthotopic neuroblastoma mouse model showed statistically significant inhibition of xenograft growth ${ }^{100}$. While these findings are encouraging, little is known about the binding modes of these compounds and whether they can be further optimized into more 'drug-like' entities. Recently, Almac Discovery and Genentech reported that fragment-based screens provided hits as starting points for USP7 discovery programmes ${ }^{101}$. Optimization of one hit, ADC-01, assisted by X-ray crystallography, produced the non-covalent, highly selective USP7 inhibitor ADC-03 (TABLE 2).

The stability of p53 has also recently been reported to be regulated by the DUB ATXN3 (REF. 102). ATXN3 was shown to bind to and deubiquitylate $\mathrm{p} 53$, resulting in p53 stabilization. Deletion of ATXN3 resulted in destabilization of p53, whereas ectopic expression of ATXN3 induced expression of p53 target genes and promoted p53-dependent apoptosis. How and whether ATXN3 inhibitors could be exploited to treat cancer or other diseases remains to be established.

USP2 8 is another DUB that has recently been connected to $\mathrm{p} 53$, which functions together with TP53-binding protein 1 (TP53BP1) to promote $\mathrm{p} 53$ mediated transcriptional responses ${ }^{103}$. Furthermore, USP28 is mutated in human cancer cells and is reported to antagonize the tumour suppressor F-box and WD40 domain-containing protein 7 (FBW7) ${ }^{31}$, highlighting the potential for USP28 inhibitors in various tumours, especially colorectal cancer ${ }^{104}$. USP2 8 has also been reported to antagonize ubiquitin-dependent degradation of the oncogene product MYC as well as JUN and Notch $^{105}$. Although no USP28 inhibitors have yet been reported, it seems likely that drug discovery activities are underway.

HIF1 $\alpha$ and USP20. Another tumour suppressor that has been linked to DUB activity is the von HippelLindau (VHL) protein, which ubiquitylates hypoxiainducible factor $1 \alpha$ (HIF1a) when cellular oxygen levels are normal, leading to the degradation of HIF1a. USP20 (also known as VDU2), is reported to deubiquitylate a number of proteins, including HIF1a.
USP20-mediated deubiquitylation of HIF1a prevents proteasomal degradation, allowing for transcription of hypoxic response genes. Thus, inhibition of USP20 has potential for suppressing proliferation of hypoxic tumour cells. GlaxoSmithKline (GSK) presented brief details of its search for USP20 inhibitors at a conference in 2012 (REF. 106) (TABLE 2).

EGFR and USP8. Ubiquitylation serves as a signal that delivers membrane receptors from the cell surface to lysosomes, and in mammalian cells, this mechanism has been most intensively studied for epidermal growth factor receptor (EGFR). Upon EGF binding, activated EGFR is rapidly internalized and transported, via early and late endosomes to lysosomes, where EGFR is degraded. USP8 (also known as UBPY) deubiquitylates EGFR on early endosomes, rescuing EGFR from degradation ${ }^{107,108}$. In several tumours - including glioblastoma, lung and breast cancer - EGFR is amplified or mutated in the tyrosine kinase domain, resulting in deregulation of receptor signalling that drives uncontrolled proliferation of tumour cells ${ }^{109}$. USP8 inhibitors (for example, HBX90659) of a similar structural class to those identified for USP7 (REF. 110) have been reported (TABLE 2). Moreover, a derivative of these compounds was shown to be efficacious in mouse models of lung cancer ${ }^{111}$

TGF $\beta$ and USP15. USP15 regulates the transforming growth factor- $\beta$ (TGF $\beta$ ) pathway and is thought to be important for the proliferation of glioblastoma cells ${ }^{22}$. USP15 binds to the SMAD7-SMAD-specific E3 ubiquitin protein ligase 2 (SMURF2) complex, and deubiquitylates and stabilizes the type I TGF $\beta$ receptor, leading to enhanced TGF $\beta$ signalling. The USP 15 gene is amplified in glioblastoma, breast and ovarian cancers, and high expression of USP15 correlates with high TGF $\beta$ activity ${ }^{22}$. Depletion of USP15 reduces the oncogenic capacity of patient-derived glioma-initiating cells owing to diminished TGF $\beta$ signalling, suggesting therapeutic potential for development of USP15 inhibitors. In addition, USP15 has been shown to deubiquitylate receptor-regulated SMADs (R-SMADs) ${ }^{112}$, another set of TGF $\beta$ signalling pathway components.

UCHL1. The DUB UCHL1, normally expressed predominantly in neurons and neuroendocrine tissues $^{113,114}$, is highly expressed in many cancers, and its expression correlates with poor prognosis ${ }^{115}$. Although there are reports that UCHL1 has a tumour-suppressive role, most evidence supports its role as an oncogene ${ }^{115}$. Indeed, in a transgenic mouse model with constitutively activated UCHL1, sporadic tumours developed in many tissues ${ }^{116}$. Moreover, in vitro tumorigenesis studies showed that UCHL1 expression stimulated oncogenesis and an invasive phenotype ${ }^{117-119}$, whereas UCHL1 depletion had antitumour effects and blocked cell migration in a lung cancer cell line ${ }^{117}$. The precise mechanism by which UCHL1 contributes to tumorigenesis remains unclear, although reports suggest that it contributes to cell survival signalling, cell cycle 
regulation, DNA repair and regulating pools of free ubiquitin in ways that affect protein degradation and function $^{115}$. UCHL1 inhibitors have been described, the most potent being isatin $O$-acyl oximes (such as LDN-57444; TABLE 2) with some selectivity over UCHL3 (REF. 120). In addition, a series of pyridinones have been identified as moderate UCHL1 inhibitors ${ }^{121}$. Enzyme kinetic studies revealed that these compounds are uncompetitive inhibitors and are selective for UCHL1, exhibiting no inhibition of other cysteine hydrolases tested. Using X-ray crystallography, a weak tripeptide fluoromethyl ketone inhibitor was subsequently shown to bind within the UCHL1 active site, irreversibly modifying the active-site cysteine ${ }^{122}$. Mission Therapeutics has also developed several series of potent and selective UCHL1 inhibitors ${ }^{123,124}$. Although no UCHL1 inhibitors have demonstrated antitumour activity in vivo, inducible depletion of UCHL1 has been shown to cause disease regression in an orthotopic multiple myeloma mouse model ${ }^{125}$.

USP22. Another DUB associated with oncogenesis is USP22, the catalytic subunit of a deubiquitylase module in the SAGA (Spt-Ada-Gcn5-acetyltransferase) complex. The best-characterized substrates for the SAGA complex include several acetylation sites in histone $\mathrm{H} 3$ and a ubiquitylation site in histone $\mathrm{H} 2 \mathrm{~B}$, post-translational modification of which regulates gene expression ${ }^{29}$. USP22 has strong links to oncogenesis ${ }^{29}$, having been identified in microarray screens as part of an 11-gene 'death-from-cancer' signature for highly aggressive, therapy-resistant tumours. USP22 was later shown to act as an oncogene product, regulating cell cycle progression, proliferation and apoptosis ${ }^{126}$. Increased expression of USP22 has been connected with poor prognosis in several cancers, including liver ${ }^{127}$, colorectal ${ }^{127}$ and breast cancers ${ }^{128}$ as well as oesophageal squamous cell carcinoma ${ }^{129}$ and oral squamous cell carcinoma ${ }^{130}$. If USP22 DUB activity can be linked to survival and progression of these cancers, then inhibitors may provide attractive prospects for new therapies.

\section{Cancer immunotherapy}

Given the role of ubiquitin modifications and DUBs in many inflammatory processes (see below), as well as the renewed interest in targeting the immune system to fight cancer, the antineoplastic potential of therapeutically inhibiting DUBs involved in the immune system is being investigated. Among these is USP7, which positively regulates the stability of FOXP3, a crucial transcription factor controlling the differentiation of regulatory $\mathrm{T}$ cells $\left(\mathrm{T}_{\mathrm{reg}}\right.$ cells $)^{34}$. In a search for DUBs that contribute to GATA3 stabilization in FOXP3-expressing cells, both USP7 and USP21 were shown to upregulate GATA3-mediated activity using a reporter assay ${ }^{131}$. Furthermore, depletion of USP21 in $\mathrm{T}_{\text {reg }}$ cells resulted in downregulation of FOXP3, compromised expression of $\mathrm{T}_{\text {reg }}$ signature genes and impaired their suppressive activity ${ }^{132}$. As $T_{\text {reg }}$ cells restrict antitumour immune responses and promote tumour survival ${ }^{133}$, these results suggest that depletion of FOXP3 in $\mathrm{T}_{\text {reg }}$ cells by targeting USP7 and USP21 offer promise for anticancer immunotherapies. In this regard, Mission Therapeutics is investigating USP7 as an immuno-oncology target and has developed USP7 inhibitors (See Mission Therapeutics pipeline in Further information).

\section{DUBs in neurodegenerative disease}

Identification of ubiquitin in protein aggregates associated with neurodegenerative pathologies - such as neurofibrillary tangles in Alzheimer disease, neuronal inclusion bodies (also known as Lewy bodies) in Parkinson disease or intranuclear inclusions in hereditary polyglutamine expansion disorders - has prompted much interest in understanding how ubiquitylation and deubiquitylation affect such aggregates ${ }^{134}$. DUB function in the central nervous system has been described in detail elsewhere ${ }^{135,136}$, therefore, below we focus on a selected number of DUBs connected to neurodegenerative disease.

\section{Mitochondrial quality control}

Mitochondrial dysfunction and UPS impairment have been described as hallmarks of ageing ${ }^{137}$ and have been implicated in the aetiopathogenesis of many age-related diseases, particularly neurodegenerative disorders such as Alzheimer disease and Parkinson disease. In accord with this connection, ubiquitylation has close links to mitochondrial function, with the UPS maintaining mitochondrial homeostasis by regulating organelle dynamics, the mitochondrial proteome and mitophagy ${ }^{138}$. Conversely, mitochondrial dysfunction can impair cellular protein homeostasis by generating oxidative damage. Notably, mutations in the ubiquitin E3 ligase Parkin are causally associated with certain cases of familial Parkinson disease ${ }^{139}$. As Parkin ubiquitylates mitochondrial components, thus promoting turnover of mitochondria by lysosome-mediated mitophagy, defective mitophagy and accumulation of defective mitochondria that cause enhanced oxidative stress could be an underlying cause of Parkinson disease ${ }^{140,141}$. A corollary of this is that Parkin activation - or inhibition of factors counteracting Parkin - could provide opportunities for disease alleviation.

A screen for DUBs that oppose Parkin function identified the mitochondrion-associated DUB USP30 as an antagonist of Parkin-mediated mitophagy ${ }^{142,143}$, with USP30 depletion significantly decreasing mitochondrial numbers in cells, a phenotype that was rescued by wildtype but not catalytically inactive USP30. Furthermore, USP30 depletion in vivo provided stress protection in Drosophila melanogaster models of Parkinson disease. In line with such findings, USP30 depletion in human HeLa cells led to elongated and interconnected mitochondria ${ }^{144}$, suggesting a role for USP30 in regulating mitochondrial fusion and fission. Current models invoke USP30 functioning under normal physiological conditions to prevent inappropriate mitophagy. However, in response to stresses such as membrane depolarization, Parkin is recruited to mitochondria to promote mitophagy ${ }^{145}$. Accordingly, under conditions of mitochondrial dysfunction - for example, caused by defects in 
Parkin (or its positive regulator PINK1) - USP30 is thought to counteract clearance of damaged mitochondria, leading to a build-up of metabolically and energetically deficient cells ${ }^{142}$. It is thus hypothesized that, in the context of certain mitochondrial dysfunctions, USP30 inhibition would have therapeutic benefits. So far, only one chemical inhibitor of USP30 has been described, 15-oxospiramilactone (TABLE 2), which induced mitochondrial elongation in $M f n 1$-knockout mouse fibroblasts, with no effect on cell viability ${ }^{146}$. Mission Therapeutics is exploring USP30 inhibition for the treatment of Parkinson disease and other mitochondrial disorders, and has published several patent applications describing USP30 inhibitors ${ }^{124,147}$.

Two other DUBs connected to mitophagy are USP8 and USP15. Notably, USP8 depletion was found to delay Parkin translocation onto depolarized mitochondria, as well as mitochondrial clearance, and USP8 displayed an ability to remove K6-linked ubiquitin chains from Parkin in vitro ${ }^{148}$. In addition, USP8 has been shown to remove K63-linked ubiquitin chains from $\alpha$-synuclein ${ }^{149}$, a protein known to aggregate - often in a ubiquitylated form - in Lewy bodies associated with neurodegenerative diseases such as Parkinson disease. Depletion of USP8 in either human SH-SY5Y cells or D. melanogaster resulted in increased lysosomal degradation of a-synuclein ${ }^{149}$. Meanwhile, USP15 was identified as a Parkin-interacting protein that colocalizes with mitochondria ${ }^{150}$. In HeLa cells overexpressing Parkin, overexpression of wild-type but not catalytically dead USP15 strongly inhibited mitophagy ${ }^{143,150}$. Furthermore, depleting endogenous USP15 enhanced mitophagy in HeLa cells, in a human dopaminergic neuronal cell line and in primary fibroblasts from human patients ${ }^{150}$. USP15 does not deubiquitylate Parkin under basal conditions or when cells are treated with mitochondrial depolarizing agents. USP15 depletion also does not seem to affect Parkin translocation to mitochondria ${ }^{150}$, although it can oppose Parkin-mediated mitochondrial ubiquitylation. Finally, loss of USP15 in D. melanogaster was found to rescue both locomotor defects and accumulation of dysfunctional mitochondria in flight muscles of parkin-knockout flies ${ }^{150}$. Collectively, these findings highlight the potential for USP8 and USP15 inhibitors in Parkinson disease and perhaps other diseases associated with mitochondrial dysfunction.

Further highlighting connections between Parkinson disease and DUBs, ATXN3 has been shown to interact with Parkin in a manner that counteracts Parkin autoubiquitylation ${ }^{151}$. In addition, USP7 was recently shown to remove K63-linked ubiquitin chains from $\alpha$-synuclein ${ }^{149}$, a protein that aggregates and accumulates in Lewy bodies, which are hallmarks of Parkinson disease. overexpressed proteins, such as Tau, TAR DNA-binding protein 43 (TDP43) and ATXN3, whose accumulation is linked to neurodegenerative diseases ${ }^{46}$. Notably, IU1 only promoted degradation in Usp $14^{+/+}$murine embryonic fibroblasts ${ }^{46}$ but not in Usp $14^{-/-}$cells, suggesting that this compound functions specifically through USP14. Furthermore, IU1 reduced accumulation of menadione-induced oxidized proteins and ameliorated menadione- or hydrogen peroxide-induced cell death in human HEK293 cells ${ }^{46}$. Proteostasis Therapeutics (in collaboration with Biogen) is developing USP14 inhibitors for the clearance of aggregation-prone proteins, including a-synuclein in Parkinson disease and Tau in Alzheimer disease (see Proteostasis pipeline in Further information) and has published several patent applications describing USP14 inhibitors ${ }^{153-155}$.

Despite the growing interest in USP14 as a therapeutic target in cancer and neurodegeneration, the fact that its loss causes severe morbidity and postnatal lethality requires further investigation, especially in regard to its role in neuromuscular junctions: the neuromuscular phenotype of USP14-deficient $a x^{\jmath}$ mice is rescued by neuronal-specific expression of USP14 (REF. 156). Furthermore, the extent to which USP14 contributes to the clearance of proteins involved in neurodegeneration in vivo remains controversial ${ }^{157}$. The development and use of USP14 inhibitors in disease-relevant models may shed further light on such issues and hopefully will define potential therapeutic windows for USP14 inhibition in disease settings.

\section{USP16}

Down syndrome is a congenital disorder driven by triplication of human chromosome 21 , on which the USP 16 gene resides. USP16 has been reported to regulate cell cycle progression and gene expression through deubiquitylation of histone $\mathrm{H}_{2} \mathrm{~A}^{158}$. Defects in haematopoietic stem cell self-renewal in a mouse model of Down syndrome were rescued by reducing USP16 expression to levels similar to those in control mice ${ }^{159}$. In addition, USP16 overexpression in normal human fibroblasts and neural progenitors led to reduced cell expansion ${ }^{159}$, which was similar to the strong proliferation defects observed in fibroblasts from individuals with Down syndrome ${ }^{160}$. Thus, USP16 is a key regulator that controls stem cell self-renewal and senescence in Down syndrome, suggesting that inhibitors of USP16 might provide therapeutic benefits to such individuals.

\section{DUBs in immunity and inflammation}

Pathogens are recognized by several families of pattern recognition receptors and activate various signal transduction cascades via the RIG-I-like receptors (RLRs), the nucleotide-binding oligomerization domain-like receptors (NLRs) and the toll-like receptors (TLRs) ${ }^{161}$. These signalling events mediate induction of inflammation that is important for recruiting immune cells to sites of infection. Ubiquitylation is a critical post-translational modification in this process ${ }^{161}$. Non-degradative K63-linked and M1-linked ubiquitin chains mediate the key upstream event of recruiting the TGF $\beta$-activated 
kinase 1 (TAK1) and the IKB kinase (IKK) complexes, respectively ${ }^{162}$. K63-linked polyubiquitylation activates the TAK1 complex, which phosphorylates the subunit IKK $\beta$ at key serine residues in the activation loop, resulting in IKK activation and transcriptional activation of target genes that include mediators of immune and inflammatory responses as well as feedback inhibitors of the NF- $\kappa B$ pathway ${ }^{163}$. Negative regulators include DUBs that cleave K63-linked and linear ubiquitin chains such as A20 (also known as TNFAIP3), CYLD and OTU domain-containing deubiquitinase with linear linkage specificity (OTULIN; also known as FAM105B and Gumby $)^{161,164,165}$

A20 is probably the best-characterized DUB linked to inflammation ${ }^{166}$. This DUB plays a key part in restricting TLR signalling and maintaining immune homeostasis through deubiquitylation of NF- $\kappa \mathrm{B}$ signalling factors such as NF- $\kappa \mathrm{B}$ essential modulator (NEMO), receptor-interacting serine/threonine-protein kinase 1 (RIPK1) and tumour necrosis factor (TNF) receptorassociated factor 6 (TRAF6) ${ }^{167}$. In addition, A20 can bind to polyubiquitin chains through its zinc finger domain, allowing for interaction with ubiquitylated NEMO. This ubiquitin-induced recruitment of A20 to NEMO is sufficient to block IKK phosphorylation by its upstream kinase TAK1, preventing NF- $\kappa B$ activation $^{168}$. Thus, A20 deficiency promotes local or systemic inflammation in vivo, underscoring why inactivating TNFAIP3 mutations have connections with both inflammatory and autoimmune syndromes ${ }^{169}$.

CYLD is another DUB known to negatively regulate ubiquitylation of RIG1 (one of the major RLRs) and RIG1-mediated interferon (IFN)-regulated gene induction $^{170,171}$. CYLD binds to RIG1 and thereby inhibits ubiquitylation and signalling functions of RIG1. CYLD also inhibits the ubiquitylation of TBK1 and IKKe, which contributes to the negative regulation of IFN responses ${ }^{171}$. Consistent with this, CYLD deficiency causes constitutive activation of TBK1 and IKKe in dendritic cells. Despite enhanced RIG1 signalling, CYLD-deficient cells and mice are more susceptible to vesicular stomatitis virus infection due to attenuated signalling and antiviral gene expression induced by IFN $\beta$, suggesting a positive role for CYLD in the regulation of type I IFN receptor function ${ }^{161}$.

M1-linked ubiquitin chains are generated by the linear ubiquitin chain assembly complex (LUBAC), which consists of haem-oxidized IRP2 ubiquitin ligase 1 (HOIL1; also known as RBCK1), HOIL1-interacting protein (HOIP; also known as RNF31) and SHANKassociated $\mathrm{RH}$ domain-interacting protein (SHARPIN). LUBAC is recruited to many immune receptors and ubiquitylates target proteins, including RIPK1, RIPK2, MYD88, interleukin-1 receptor-associated kinases (IRAKs) and NEMO ${ }^{172,173}$. Genetic loss of LUBAC components leads to immunodeficiency ${ }^{174}$ and inflammatory phenotypes in mice ${ }^{175-178}$, and mutations in LUBAC components also cause inflammatory conditions in humans ${ }^{179,180}$. Hence, loss of M1-linked ubiquitin chains imbalances immune signalling. OTULIN is the only DUB known to specifically cleave M1 linkages ${ }^{181,182}$. Accordingly, a homozygous hypomorphic mutation in human OTULIN has recently been shown to cause a potentially fatal auto-inflammatory condition termed OTULIN-related autoinflammatory syndrome ${ }^{183}$. Similar to ubiquitin, the UBL IFN-stimulated gene 15 (ISG15) has a key role in cellular signalling in response to pathogens. Conjugation of ISG15 to various cellular substrates is reversed by the IFN-inducible isopeptidase USP18. USP18 is upregulated after viral infection, type I and type III IFNs, lipopolysaccharide, TNFa or genotoxic stress. In addition to its isopeptidase activity, USP18 negatively regulates type I and type III IFN signalling by blocking the IFNAR2 subunit of the interferon receptor ${ }^{184}$.

\section{Inflammatory and autoimmune disorders}

Debilitating autoimmune diseases range from those with genetic components - such as Crohn's disease, diabetes mellitus type 1, Graves disease and rheumatoid arthritis ${ }^{185}$ - to sporadic conditions, including coeliac disease, inflammatory bowel disease, multiple sclerosis, psoriasis and systemic lupus erythematosus. In addition, chronic inflammatory diseases are characterized by a prolonged and persistent pro-inflammatory state, and include autoimmune disease as well as metabolic syndrome, neurodegenerative disease, chronic obstructive pulmonary disease and cardiovascular disease.

Following pattern recognition receptor stimulation, dendritic cells secrete various cytokines that regulate the differentiation of $\mathrm{CD} 4^{+} \mathrm{T}$ cells to different subsets of $\mathrm{T}$ helper cells $\left(\mathrm{T}_{\mathrm{H}}\right.$ cells), including inducible $\mathrm{T}_{\text {reg }}$ cells, $\mathrm{T}$ follicular helper cells, and $\mathrm{T}_{\mathrm{H}} 1, \mathrm{~T}_{\mathrm{H}} 2, \mathrm{~T}_{\mathrm{H}} 9$ and $\mathrm{T}_{\mathrm{H}} 17$ cells ${ }^{186} \cdot \mathrm{T}_{\mathrm{H}} 17$ cells mediate pro-inflammatory functions through the secretion of pro-inflammatory cytokines, including interleukin-17A (IL-17A), IL-17F, and IL-22 (REF. 187). Moreover, $T_{H} 17$ cells have been implicated in the development of autoimmune diseases such as multiple sclerosis, rheumatoid arthritis and systemic lupus erythematosus $^{188}$.

USP4 has been shown to stabilize the nuclear receptor retinoid-related orphan receptor- $\gamma \mathrm{t}(\mathrm{ROR} \gamma \mathrm{t})$ in $\mathrm{T}_{\mathrm{H}} 17$-activated $\mathrm{T}$ cells and has been proposed as a possible therapeutic target for rheumatoid arthritis ${ }^{189}$. One report showed that USP4 is highly expressed in $\mathrm{T}_{\mathrm{H}} 17$ cells, and its depletion resulted in decreased ROR $\gamma \mathrm{t}$ as well as IL-17A expression ${ }^{189}$. In addition, use of the reported USP4 inhibitor vialinin A (TABLE 2) also diminished ROR $\gamma t$ and IL-17A expression ${ }^{190}$. Furthermore, expression of USP4, IL-17A and IL-17F mRNA have been shown to be significantly elevated in $\mathrm{CD} 4{ }^{+} \mathrm{T}$ cells from patients with rheumatoid arthritis compared with healthy controls ${ }^{189}$, providing further evidence for a role of USP 4 in rheumatoid arthritis.

TRABID (also known as ZRANB1) is required for TLR-mediated expression of the inflammatory cytokines IL-12 and IL-23 in dendritic cells ${ }^{191}$. It has been proposed that TRABID deubiquitylates and stabilizes the histone demethylase Jumonji domain-containing protein 2D (JMJD2D; also known as KDM4D), which regulates histone modification at the Il12 and Il23 promoters to facilitate recruitment of the NF- $\kappa B$ family member $\mathrm{REL}^{191}$. Conditional deletion of Zranb1 in dendritic cells 
impairs IL-12 and IL-23 production and the generation of $\mathrm{T}_{\mathrm{H}} 1$ and $\mathrm{T}_{\mathrm{H}} 17$ subsets of inflammatory $\mathrm{T}$ cells, rendering mice refractory to the induction of experimental autoimmune encephalomyelitis (EAE) ${ }^{191}$.

Another DUB associated with the activity of $\mathrm{T}_{\mathrm{H}} 17$ cells is USP18. Although this DUB has been extensively studied in the context of viral infection, USP18 regulates the TAK1-TAB interaction, which is required for $\mathrm{T}_{\mathrm{H}} 17$ cell differentiation and the autoimmune response $\mathrm{e}^{192}$. Consistent with this, USP18-deficient mice were resistant to $\mathrm{EAE}^{192}$.

T cell receptor signalling has been shown to be facilitated by the DUB cellular zinc finger anti-NF- $\kappa \mathrm{B}$ protein (CEZANNE; also known as OTUD7B), which binds to and deubiquitylates $\zeta$-chain-associated protein (ZAP70), thus preventing the interaction of ZAP70 with negative regulatory phosphatases ${ }^{193}$. ZAP70 is a cytoplasmic protein tyrosine kinase that plays a crucial part in $\mathrm{T}$ cell signalling and is recruited to phosphorylated sites on the T cell receptor, where it is subsequently phosphorylated by the SRC kinase LCK. Phosphorylation of ZAP70 is required for full activation and downstream phosphorylation of adaptor proteins, which facilitate $\mathrm{T}$ cell signalling ${ }^{194}$. In addition, CEZANNE-deficient mice exhibited attenuated $\mathrm{T}$ cell responses to bacterial infection and were refractory to $\mathrm{EAE}^{193}$. While young Otud $7 b$-knockout mice had similar naive and memorylike $\mathrm{T}$ cells compared with wild-type mice, older mice deficient for CEZANNE had reduced IFN $\gamma$-producing $\mathrm{T}_{\mathrm{H}} 1$ cell subsets ${ }^{193}$.

Similar to $T_{H} 17$ cells, $T_{H} 1$ cells have the capacity to cause inflammation and autoimmune disease. The development, differentiation and function of $\mathrm{T}_{\mathrm{H}} 1$ cells is driven by the T-box transcriptional factor T-bet (also known as TBX21), which promotes $\mathrm{T}_{\mathrm{H}} 1$-mediated immune response primarily through promoting expression of the cytokine IFN $\gamma^{195}$. The DUB USP10 has been shown to deubiquitylate and stabilize T-bet, resulting in enhanced secretion of IFN $\gamma^{196}$. In addition, USP10 mRNA expression was found to be elevated in peripheral blood mononuclear cells from patients with asthma compared with healthy donors ${ }^{196}$.

Although it is currently unclear why so many DUBs are involved in the regulation of immune responses, it is possible that different DUBs function in distinct cell types. Many published studies are based on cell lines and overexpression systems, and the expression of endogenous DUBs in various immune cells will be an important area for future investigation. Similarly, the generation of genetic models and the development of inhibitors for CEZANNE, TRABID, USP4, USP10 and USP18 will help determine their therapeutic potential.

\section{DUBs in infectious diseases}

As described below, there is growing interest in DUBs as potential therapeutic targets for various infectious diseases of man and other animals. Such potential is being explored both by developing compounds that inhibit the activity of pathogen-encoded DUB-like proteins, or target host cell DUBs that control the pathogen life cycle or infectivity.

\section{Viral infections}

Ubiquitylation is important for modulation of protein-protein interactions, including the activation of innate immune signalling pathways, so perhaps it is not surprising that various viruses have genes for DUBs, which are used as a strategy to inhibit ubiquitin- and ISG15-dependent antiviral pathways ${ }^{197}$. Severe acute respiratory syndrome coronavirus (SARS-CoV) and Middle East respiratory syndrome coronavirus (MERS$\mathrm{CoV}$ ) are two of the six known human coronaviruses. Both are highly pathogenic, with the potential for human-to-human transmission, and contain papain-like cysteine proteases termed SARS-CoV PLpro and MERSCoV PLpro, respectively. In addition to processing viral polyprotein, these proteases remove ubiquitin and ISG15 from host cell factors, resulting in antagonism of the host antiviral immune response ${ }^{198}$. Hence, both SARS-CoV PLpro and MERS-CoV PLpro have been proposed as important antiviral targets. The X-ray structures of both proteases have shown structural similarity to the USP family of DUBs ${ }^{199-201}$.

OTU domain-containing proteases from diverse RNA viruses, including the nairoviruses CrimeanCongo haemorrhagic fever virus and Dugbe virus, the papain-like protease (PLP2) domain of the arterivirus equine arteritis virus, and the protease (PRO) domain of the tymovirus turnip yellow mosaic virus can hydrolyse ubiquitin and ISG15 from cellular target proteins ${ }^{197,202}$. Many positive-strand RNA viruses, including arteriviruses and tymoviruses, encode polyproteins that are posttranslationally cleaved by internal protease domains. In accord with this, both arterivirus PLP2- and tymovirus PRO-domain-containing proteases are crucially required for viral replication due to their primary role in polyprotein maturation ${ }^{197}$. Thus, viral OTU domain-containing proteases may represent promising therapeutic targets.

\section{Bacterial infections}

Bacteria use a repertoire of effector proteins that target the eukaryotic ubiquitin system to promote bacterial pathogenicity. Protease activities from human bacterial pathogens, including Salmonella enterica serovar Typhimurium (SseL), Escherichia coli (ElaD), Shigella flexneri (ShiCE), Chlamydia trachomatis (ChlaDUB1), Rickettsia bellii (RickCE) and Legionella pneumophila subsp. pneumophila (LegCE), have recently been characterized ${ }^{203}$. LegCE showed no proteolytic activity; SseL, ElaD, and ShiCE demonstrated ubiquitin-specific protease activity; whereas ChlaDUB1 and RickCE cleaved both ubiquitin and, to a lesser extent, NEDD8-modified peptides. Interestingly, these DUBs encoded by human pathogens showed strong preference for K63-linked ubiquitin chains, only targeting K48-linked and K11-linked chains at later time points or higher enzyme concentrations. Therefore, bacterial DUBs are potential therapeutic targets.

\section{Parasitic infections}

In addition to expressing DUBs that target host functions, similar to viruses and bacteria, eukaryotic parasites also possess UBL pathways of their own. The use of ubiquitin-based activity probes to identify DUBs 
in Plasmodium falciparum led to the identification of PfUCH54, which was shown to have deubiquitylating activity and also an ability to remove adducts of the UBL NEDD8 (REF. 204). Further investigation of the parasite Toxoplasma gondii using a similar strategy identified four DUBs, one of which was orthologous to mammalian UCHL3 (REF. 205). Structural studies on PfUCHL3 explained the dual specificity of the enzyme, and PfUCHL3 was found to be required for parasite survival ${ }^{206}$. Distinct differences in the ubiquitin-binding site between PfUCHL3 and its human counterpart suggest that this parasitic DUB can be selectively targeted by inhibitors. Based on the above findings, it will be of great interest to further explore anti-infective opportunities for DUB inhibitors.

\section{Challenges and emerging technologies}

Despite the significant and growing attractiveness of DUBs as drug targets, DUB-focused drug discovery has been challenging, with researchers in this arena facing various obstacles. First, although DUBs have clear catalytic pockets that a priori seem suitable for drug development, a key challenge has been to identify potent compounds that show selectivity among related DUBs and that have properties commensurate with their development for clinical use. Second, ubiquitylation and deubiquitylation are intracellular processes that, at least at present, are only amenable to classical small-molecule chemical approaches. Third, because most DUBs execute the transfer of ubiquitin molecules via a reactive thiol group, most standard assays used to identify inhibitors are prone to non-selective redox or alkylating false positives ${ }^{207}$. Fourth, the mechanisms of action of DUB enzymes are often complex, involving regulation of enzymatic activity through allosteric effects and/or substrate-mediated catalysis, and many DUBs alternate between active and non-active conformations (see below) ${ }^{208,209}$. This makes it challenging both to design predictive biochemical assays and develop drug-like compounds. Finally, DUBs often display specificity for ubiquitin chains as well as the target proteins. Hence, to optimize the likelihood of identifying genuine inhibitors, it is prudent to develop bespoke primary screening and secondary assays that recapitulate the most physiological substrate and ubiquitin-linkage setting for each DUB.

Despite the above issues, DUBs are fundamentally catalytically driven proteins with known enzymatic functions and, as such, present researchers with the opportunity to identify small-molecule inhibitors either within the active site or at adjacent allosteric pockets. Indeed, over the past few years there has been an increasing rate of progress in successfully screening for and evolving small-molecule DUB inhibitors, with the most developed ones now moving towards or into clinical evaluation (for examples, see TABLE 2).

Allosteric

Binding to a site other than

the active site of the enzyme, resulting in modulation of enzymatic activity. proteolytic reaction between a lysine $\varepsilon$-side chain and a carboxyl group corresponding to the ubiquitin $\mathrm{C}$ terminus ${ }^{209}$. The last two C-terminal amino acid residues are glycines (Gly75-Gly76) that lack side chains, resulting in a narrow linker on either side of the isopeptide bond, which is mirrored in a long and narrow DUB catalytic cleft ${ }^{209}$. Moreover, cysteinyl protease DUB catalytic activity tends to rely on two or three crucial residues comprising a catalytic diad or triad, generally containing a His side chain that - by lowering the $\mathrm{p} K_{\mathrm{a}}$ of the catalytic Cys - leads to a nucleophilic attack on the ubiquitinsubstrate isopeptide linkage ${ }^{12}$. Collectively, these properties bring complexity to the identification of selective small-molecule inhibitors that target DUB catalytic sites and are likely to restrict the breadth of series that are suitable for developing potent and selective DUB inhibitors.

The Proteostasis thiophene pyrimidine core-derived USP14 inhibitors are known to bind in the ubiquitin pocket and prevent the ubiquitylated substrate from binding ${ }^{210}$. However, the majority of historical and current DUB drug discovery programmes have focused on chemical series that include the provision of an active 'warhead' that forms a reversible or irreversible covalent adduct with the DUB catalytic cysteine. The high reactivity of some of these warheads, which include oxidative, alkylating and arylating moieties ${ }^{210}$, is likely to limit drug selectivity, may hamper the development of acceptable pharmacokinetic and pharmacodynamic parameters, and may also pose risks of idiosyncratic toxicities in patients.

For this reason, less-reactive warheads are being explored that are closely related to warheads utilized by non-DUB cysteine protease inhibitors in the clinic. For example, the USP8 inhibitor identified from a library of

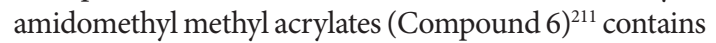
a Michael acceptor group also found in rupintrivir, an inhibitor of rhinovirus 3C protease and a GSK cathepsin C inhibitor ${ }^{210}$. In addition, USP9X inhibitors WP1130 and EOA1342143 (REF. 212) contain a Michael acceptor group similar to that found in a Principia Biopharma's Bruton tyrosine kinase (BTK) inhibitor ${ }^{210}$. However, these examples are few in number, and the compounds are weak DUB inhibitors. Mission Therapeutics has discovered covalent active-site series that are 'drug-like', unrelated to any previously described DUB inhibitor, and that achieve submicromolar cell-based potencies and exhibit good oral bioavailability ${ }^{123,124,147}$.

\section{Allosteric regulation: implications}

Most peptidases, including many cysteine proteases, recognize a small linear-polypeptide motif and cleave either before or after the peptide bond ${ }^{213}$. DUBs, however, are more complex. Most DUBs cleave an isopeptide linkage between the side chain of a lysine residue and the C-terminal glycine of ubiquitin, with the isopeptide linkage providing specificity and flexibility to the mechanism of proteolysis ${ }^{214}$. Also, DUBs need to accommodate substantial globular post-translational modifications (ubiquitin, UBL, or ubiquitin-UBL chains) into their catalytic site ${ }^{215}$. Furthermore, unlike most other cysteine peptidases, the catalytic triad of cysteinyl peptidase DUBs is not usually in a 'functional' configuration, 
with allosteric regulation being required to render DUBs fully functional and processive. Such allosteric regulation can be substrate-mediated (for example, OTULIN) ${ }^{181}$, triggered by intramolecular reorganization (for example, USP7) ${ }^{216}$ or induced by key cofactors (as for USP1) ${ }^{74}$. In addition, several DUBs are associated with multi-protein complexes such as the proteasome ${ }^{217}$, valosin-containing protein (VCP; also known as p97) ${ }^{218}$, or the COP9 signalosome ${ }^{219}$. These associations can allosterically regulate the affinity of DUBs for their substrates ${ }^{208,220}$, and in some instances, DUBs coexist in the same complex as the ubiquitylation machinery ${ }^{221}$. The above issues must therefore be carefully considered when establishing screening and compound evaluation assays for a DUB. Some DUB inhibitors have been suggested to target allosteric sites, such as the USP1 inhibitor ML323 (REF. 78).

\section{Screening technologies}

Approximately twenty years ago, a general assay was established for measuring DUB enzymatic activity based on the substrate ubiquitin C-terminal 7-amido-4methylcoumarin (Ub-AMC). This substrate is efficiently cleaved or hydrolysed by various DUBs, releasing a highly fluorescent AMC moiety. While this assay has been used in various DUB inhibitor screens, for example to identify USP1 (REF. 222) and USP7 (REFS 207,223,224) inhibitors, one significant drawback is that it is prone to fluorescence interference exhibited by many small molecules ${ }^{225}$. Moreover, AMC and alternative tags such as rhodamine and tetramethylrhodamine (TAMRA), which have been used because they are less prone to fluorescence artefacts, contain a peptide linkage and thus differ quite substantially from most natural DUB substrates. Processing of such substrates thus requires the DUB to function in a non-physiological manner, thereby potentially diminishing prospects for identifying compounds that will operate in cellular or therapeutic settings.

A further challenge for development of DUB inhibitor screening assays is oxidative hydrolysis of the active-site cysteinyl residue of purified DUBs in biochemical buffers. This sensitivity requires use of protective reducing agents such as dithiothreitol (DTT), usually in millimolar concentrations, to maintain DUB enzymatic activity. Altering the concentration or type of reducing agent (for example, 2-mercaptoethanol, cysteine, glutathione or tris(2-carboxyethyl)phosphine (TCEP)) can considerably affect inhibition obtained for hit compounds ${ }^{207}$. Following a high-throughput screen to identify USP7 inhibitors, the ability of compounds to inhibit USP7 in the presence of different reductants was evaluated ${ }^{207}$. Many compounds showed the greatest inhibition in the absence of any reductant, being less potent in the presence of cysteine or glutathione, and least potent in the presence of DTT or TCEP. A further subset of molecules showed an alternative profile, only demonstrating inhibition in the presence of DTT or TCEP. A final set of molecules only inhibited USP7 when no additional reductant was added. Together, these data demonstrate the critical nature of the reducing environment on DUB activity and inhibition. Thus, most screens based on high concentrations of reducing agents and using first-generation fluorescent substrates generate high false-positive rates, an issue that has likely been the most significant challenge in identifying genuine and selective DUB inhibitors.

Indeed, the non-selective nature of some DUB inhibitors is highlighted in biochemical selectivity-profiling assays, with relatively few DUB inhibitors reported in the literature showing promise in such studies ${ }^{76}$. Matrixassisted laser desorption/ionization time-of-flight (MALDI-TOF) mass spectrometry was used to screen for DUB activity and specificity by systematically assessing the specificities of 42 recombinant human DUBs against di-ubiquitin isomers with all possible chain linkages (M1- (linear), K6-, K11-, K27-, K29-, K33-, K48- and K63-linked) $)^{76}$. Subsequently, a panel of 32 DUBs was screened against 9 reported DUB inhibitors. Their findings demonstrated that none of the compounds displayed strong selectivity towards a single DUB and that many inhibited most DUBs on the panel.

Novel technologies based on chemically synthesized DUB substrates containing isopeptide linkages, ubiquitin chains and/or assay technologies that are less prone to false positives - such as luminescence, time-resolved fluorescence or mass spectrometry - are advancing screening campaigns and are therefore now being exploited ${ }^{76,226-228}$. For example, a ubiquitin-aminoluciferin substrate was used with a variety of DUBs to demonstrate a suitable assay window for high-throughput screening ${ }^{207,229}$. Subsequently, USP2 was used as a representative DUB to demonstrate statistical robustness of this reagent in a screening campaign for inhibitors. We believe that such developments are crucial to optimize the prospects for identifying and developing DUB inhibitors for ultimate clinical use.

\section{Monitoring DUB activity or inhibition}

A key issue when studying DUBs and their modulation in cells is understanding substrate specificity. Some DUBs have preferences for monoubiquitylated substrates, whereas others favour specific ubiquitin chain types, chains bearing mixed linkages, or mixed chains containing ubiquitin and UBLs ${ }^{230,231}$. Furthermore, many DUBs have some specificity for the substrate protein itself, with this being mediated through mechanisms often involving regions of the DUB distinct from its catalytic site. DUB substrates can be determined by biochemistry, yeast two-hybrid interactions, proteomic profiling and genet$\mathrm{ics}^{232}$, but this is often challenging and time-consuming. Clearly, the ability to directly monitor DUB activity within a native biological system is essential to understanding the physiological and pathological role of individual DUBs as well as the effects of DUB inhibition ${ }^{233}$.

DUB activity in cells can be monitored by chemical probes that generate readily detectable covalent complexes with the DUB catalytic site (recently reviewed in REF. 234). Activity probes label DUBs based on their catalytic-site thiol group ${ }^{235}$, with DUB reactivity towards such probes depending on the type of electrophilic warhead fused to ubiquitin. In addition to profiling DUB levels, activity and catalytic inhibition, activity probes have also been used to identify DUBs by affinity purification combined with mass spectrometry ${ }^{236}$. More recently, activity-based probes (ABPs) bearing a fluorescent reporter tag have 
been generated to replace the initial tags (for example, the haemagglutinin (HA) epitope) to allow fluorescent imaging instead of detection by immunoblotting ${ }^{226,227}$. Although production of ubiquitin ABPs was historically based on a trypsin-catalysed transpeptidation to modify ubiquitin at its $\mathrm{C}$ terminus with a vinyl sulfone group, recent approaches involve the full chemical synthesis of ubiquitin $\mathrm{ABPs}^{226,237}$. This advance allows incorporation of modified amino acid residues at any position in the ABPs, whether natural or not. Mass spectrometry has become an important tool to monitor ubiquitin adducts as well as changes in ubiquitin levels ${ }^{232,238}$. Indeed, combining ABPs with immunoblotting or mass spectrometry can generate powerful tools for monitoring DUB activity and inhibition by small molecules ${ }^{98,239}$ as well as assessing drug-enzyme target engagement in cells or tissues. For example, one study used ABPs to demonstrate the selectivity of P22077 for USP7 in cells, in contrast to PR-619, which inhibited a broad range of $\mathrm{DUBs}^{239}$. In addition, another study demonstrated the cellular selectivity of HBX19818 for USP7 against a panel of DUBs using ABPs and immunoblotting ${ }^{98}$.

Activity-based proteomic probes have facilitated the development of pharmacologically active enzyme inhibitors. This approach represents a cell-based assay in which treatment with the inhibitor is carried out in intact cells, allowing for a range of cellular enzymes to be assessed simultaneously ${ }^{239}$. Competition assays between an inhibitor and the ABP lead to a reduced labelling profile for the ABP, with loss of signal for ABP-labelled target enzymes allowing for assessment of the specificity of inhibition.
The limitation to this approach, however, is the number of enzymes successfully labelled by the ABP and the representation of active enzymes in the cellular proteome. ABPs were used to characterize the DUB inhibitors PR-619 and P22077 by immunoprecipitation, combined with identification and label-free quantification by mass spectrometry-based proteomics ${ }^{239}$. Using this approach, quantitative data for 25 cellular DUBs was obtained. PR-619 was confirmed as a broad DUB inhibitor, whereas P22077 was found to be a selective inhibitor of USP7 and USP47 that may therefore provide the basis for exploring therapeutic opportunities in oncology (see preceding sections and TABLE 2).

\section{Concluding remarks}

During the past decade, we have witnessed dramatic advances in our understanding of DUB functions, mechanisms of action, regulation and disease linkages. In parallel, there have been major improvements in DUB biochemical assays and screening technologies, leading to the development of increasing numbers of small-molecule DUB inhibitors whose selectivity is now being explored and, where possible, refined. Such inhibitors are providing the basis for drug-like molecules suitable for clinical evaluation and are also providing versatile tools to further investigate DUB cell biology, regulation and biochemical mechanisms, as well as to test therapeutic hypotheses in disease models. Although it is still too early to predict the extent of the broad therapeutic potential of DUBs, the next few years certainly seem set to produce further exciting developments in the arenas of DUB biology and drug discovery.
1. Ciechanover, A. The ubiquitin proteolytic system and pathogenesis of human diseases: a novel platform for mechanism-based drug targeting. Biochem. Soc. Trans. 31, 474-481 (2003).

2. Ciechanover, A. Proteolysis: from the lysosome to ubiquitin and the proteasome. Nat. Rev. Mol. Cell Biol. 6, 79-87 (2005).

3. Gallastegui, N. \& Groll, M. The $26 \mathrm{~S}$ proteasome: assembly and function of a destructive machine. Trends Biochem. Sci. 35, 634-642 (2010).

4. Finley, D., Chen, X. \& Walters, K. J. Gates, channels, and switches: elements of the proteasome machine. Trends Biochem. Sci. 41, 77-93 (2016).

5. Peth, A., Besche, H. C. \& Goldberg, A. L. Ubiquitinated proteins activate the proteasome by binding to Usp 14/Ubp6, which causes 20S gate opening. Mol. Cell 36, 794-804 (2009).

6. Muratani, M. \& Tansey, W. P. How the ubiquitinproteasome system controls transcription. Nat. Rev. Mol. Cell Biol. 4, 192-201 (2003).

7. Jesenberger, V. $\&$ Jentsch, S. Deadly encounter: ubiquitin meets apoptosis. Nat. Rev. Mol. Cell Biol. 3, 112-121 (2002)

8. Hicke, L. Protein regulation by monoubiquitin. Nat. Reviews Molecular Cell Biology 2, 195-201 (2001).

9. Jackson, S. P. \& Durocher, D. Regulation of DNA damage responses by ubiquitin and SUMO. Mol. Cell 49, 795-807 (2013).

This review highlights how ubiquitylation and related processes control many cellular responses to DNA damage

10. Husnjak, K. \& Dikic, I. Ubiquitin-binding proteins: decoders of ubiquitin-mediated cellular functions. Annu. Rev. Biochem. 81, 291-322 (2012).

11. Herhaus, L. $\&$ Dikic, I. Expanding the ubiquitin code through post-translational modification. EMBO Rep. 16, 1071-1083 (2015)

12. Komander, D., Clague, M. J. \& Urbe, S. Breaking the chains: structure and function of the deubiquitinases. Nat. Rev. Mol. Cell Biol. 10, 550-563 (2009).
13. van der Veen, A. G. $\&$ Ploegh, H. L. Ubiquitin-like proteins. Annu. Rev. Biochem. 81, 323-357 (2012).

14. Huang, C. J., Wu, D., Khan, A. F.. \& Huo, L. J. DeSUMOylation: an important therapeutic target and protein regulatory event. DNA Cell Biol. 34, 652-660 (2015).

15. Murali, R., Wiesner, T. \& Scolyer, R. A. Tumours associated with BAP1 mutations. Pathology 45 116-126 (2013)

16. Oliveira, A. M. \& Chou, M. M. USP6-induced neoplasms: the biologic spectrum of aneurysmal bone cyst and nodular fasciitis. Hum. Pathol. 45, 1-11 (2014).

17. Hao, Y. H. et al. USP7 acts as a molecular rheostat to promote WASH-dependent endosomal protein recycling and is mutated in a human neurodevelopmental disorder. Mol. Cell 59, 956-969 (2015).

18. Ma, Z. Y. et al. Recurrent gain-of-function USP8 mutations in Cushing's disease. Cell Res. 25 306-317 (2015).

19. Reincke, M. et al. Mutations in the deubiquitinase gene USP8 cause Cushing's disease. Nat. Genet. 47 , 31-38 (2015)

References 18 and 19 are key publications identifying mutations in USP8 that enhance EGFR signalling and cause Cushing disease.

20. Homan, C. C. et al. Mutations in USP9X are associated with X-linked intellectual disability and disrupt neuronal cell migration and growth. Am. J. Hum. Genet. 94, 470-478 (2014).

21. Murtaza, M., Jolly, L. A., Gecz, J. \& Wood, S. A. La FAM fatale: USP9X in development and disease. Cell. Mol. Life Sci. 72, 2075-2089 (2015).

22. Eichhorn, P. J. et al. USP15 stabilizes TGF-beta receptor I and promotes oncogenesis through the activation of TGF-beta signaling in glioblastoma. Nat. Med. 18, 429-435 (2012).

23. Bignell, G. R. et al. Identification of the familial cylindromatosis tumour-suppressor gene. Nat. Genet. 25, 160-165 (2000).
24. Kawaguchi, Y. et al. CAG expansions in a novel gene for Machado-Joseph disease at chromosome 14q32.1. Nat. Genet. 8, 221-228 (1994).

25. McDonell, L. M. et al. Mutations in STAMBP, encoding a deubiquitinating enzyme, cause microcephalycapillary malformation syndrome. Nat. Genet. $\mathbf{4 5}$ 556-562 (2013).

26. Cohen, P. \& Tcherpakov, M. Will the ubiquitin system furnish as many drug targets as protein kinases? Cell 143, 686-693 (2010). This is a key perspective highlighting DUBs as attractive drug targets.

27. Huang, X. \& Dixit, V. M. Drugging the undruggables: exploring the ubiquitin system for drug development. Cell Res. 26, 484-498 (2016). This recent review highlights drug discovery efforts targeting the ubiquitin system, including DUBs.

28. Sacco, J. J., Coulson, J. M. Clague, M. J. \& Urbe, S. Emerging roles of deubiquitinases in cancer-associated pathways. IUBMB Life 62, 140-157 (2010).

29. Wang, L. \& Dent, S. Y. Functions of SACA in development and disease. Epigenomics 6, 329-339 (2014).

30. Nicholson, B. \& Suresh Kumar, K. G. The multifaceted roles of USP7: new therapeutic opportunities Cell Biochem. Biophys. 60, 61-68 (2011).

31. Cremona, C. A., Sancho, R., Diefenbacher, M. E. \& Behrens, A. Fbw7 and its counteracting forces in stem cells and cancer: Oncoproteins in the balance. Semin. Cancer Biol. 36, 52-61 (2016).

32. D’Arcy, P., Wang, X. \& Linder, S. Deubiquitinase inhibition as a cancer therapeutic strategy. Pharmacol. Ther. 147, 32-54 (2015).

33. Souroullas, G. P. \& Sharpless, N. E. Stem cells: Down's syndrome link to ageing. Nature 501, 325-326 (2013).

34. van Loosdregt, J. et al. Stabilization of the transcription factor Foxp3 by the deubiquitinase USP7 increases Treg-cell-suppressive capacity. Immunity 39, 259-271 (2013)

First report demonstrating that USP7 deubiquitylates and stabilizes FOXP3 in $T_{\text {reg }}$ cells. 
35. Sun, H. et al. Bcr-Abl ubiquitination and Usp9x inhibition block kinase signaling and promote CML cell apoptosis. Blood 117, 3151-3162 (2011).

36. Savio, M. G. et al. USP9X controls EGFR fate by deubiquitinating the endocytic adaptor Eps 15. Curr. Biol. 26, 173-183 (2016).

37. Richardson, P. G., Hideshima, T. \& Anderson, K. C. Bortezomib (PS-341): a novel, first-in-class proteasom inhibitor for the treatment of multiple myeloma and other cancers. Cancer Control 10, 361-369 (2003).

38. Chen, D., Frezza, M., Schmitt, S., Kanwar J. \& Dou, Q. P. Bortezomib as the first proteasome inhibitor anticancer drug: current status and future perspectives. Curr. Cancer Drug Targets 11, 239-253 (2011).

39. Yao, T. \& Cohen, R. E. A cryptic protease couples deubiquitination and degradation by the proteasome. Nature 419, 403-407 (2002)

40. Song, Y. et al. Deubiquitylating enzyme Rpn $11 / \mathrm{POH} 1 /$ PSMD 14 As therapeutic target in multiple myeloma. Blood 128, 4469-4469 (2016).

41. Wang, B. et al. $\mathrm{POH} 1$ deubiquitylates and stabilizes E2F1 to promote tumour formation. Nat. Commun. 6 8704 (2015)

42. Liu, H., Buus, R., Clague, M. J. \& Urbe, S. Regulation of ErbB2 receptor status by the proteasomal DUB POH1. PLOS ONE 4, e5544 (2009).

43. Kakarougkas, A. et al. Co-operation of BRCA 1 and $\mathrm{POH} 1$ relieves the barriers posed by 53BP1 and RAP80 to resection. Nucleic Acids Res. 41, 10298-10311 (2013).

44. Hu, M. et al. Structure and mechanisms of the proteasome-associated deubiquitinating enzyme USP14. EMBO J 24, 3747-3756 (2005).

45. Eletr, Z. M. \& Wilkinson, K. D. Regulation of proteolysis by human deubiquitinating enzymes. Biochim. Biophys. Acta 1843, 114-128 (2014)

46. Lee, B. H. et al. Enhancement of proteasome activity by a small-molecule inhibitor of USP14. Nature 467 , 179-184 (2010)

47. Wu, N. et al. Over-expression of deubiquitinating enzyme USP 14 in lung adenocarcinoma promotes proliferation through the accumulation of betacatenin. Int. J. Mol. Sci. 14, 10749-10760 (2013)

48. Wang, Y. et al. Ubiquitin-specific protease 14 (USP14) regulates cellular proliferation and apoptosis in epithelial ovarian cancer. Med. Oncol. 32, 379 (2015).

49. Xu, D. et al. Phosphorylation and activation of ubiquitin-specific protease- 14 by Akt regulates the ubiquitin-proteasome system. eLife 4, e 10510 (2015).

50. Jung, H. et al. Deubiquitination of Dishevelled by Usp 14 is required for Wnt signaling. Oncogenesis 2, e64 (2013).

51. Qiu, X. B. et al. hRpn13/ADRM1/GP110 is a novel proteasome subunit that binds the deubiquitinating enzyme, UCH37. EMBO J. 25, 5742-5753 (2006).

52. Yao, T. et al. Proteasome recruitment and activation of the Uch37 deubiquitinating enzyme by Adrm 1. Nat. Cell Biol. 8, 994-1002 (2006).

53. Koulich, E., Li, X. \& DeMartino, G. N. Relative structura and functional roles of multiple deubiquitylating proteins associated with mammalian 26S proteasome. Mol. Biol. Cell 19, 1072-1082 (2008)

54. Lam, Y. A., Xu, W., DeMartino, G. N. \& Cohen, R. E. Editing of ubiquitin conjugates by an isopeptidase in the 26S proteasome. Nature 385, 737-740 (1997)

55. Mazumdar, T. et al. Regulation of NF-kappaB activity and inducible nitric oxide synthase by regulatory particle non-ATPase subunit 13 (Rpn13). Proc. Nat Acad. Sci. USA 107, 13854-13859 (2010)

56. Wang, L et al. High expression of $\mathrm{UCH} 37$ is significantly associated with poor prognosis in human epithelial ovarian cancer. Tumour Biol. 35, 11427-11433 (2014)

57. Fang, Y. et al. Ubiquitin C-terminal hydrolase 37 a novel predictor for hepatocellular carcinoma recurrence, promotes cell migration and invasion via interacting and deubiquitinating PRP19. Biochim Biophys. Acta 1833, 559-572 (2013).

58. Richardson, P. G. A review of the proteasome inhibitor bortezomib in multiple myeloma. Expert Opin. Pharmacother. 5, 1321-1331 (2004).

59. Wang, X. et al. Synthesis and evaluation of derivatives of the proteasome deubiquitinase inhibitor b-AP15. Chem. Biol. Drug Design 86, 1036-1048 (2015).

60. D'Arcy, P. et al. Inhibition of proteasome deubiquitinating activity as a new cancer therapy Nat. Med. 17, 1636-1640 (2011)

61. Berndtsson, M. et al. Induction of the lysosomal apoptosis pathway by inhibitors of the ubiquitinproteasome system. Int. J. Cancer 124, 1463-1469 (2009).
62. Tian, Z. et al. A novel small molecule inhibitor of deubiquitylating enzyme USP 14 and UCHL5 induces apoptosis in multiple myeloma and overcomes bortezomib resistance. Blood 123, 706-716 (2014).

63. Zhou, H.-J. et al. Compositions and methods for JAMM protein inhibition. WO2012158435 (A1) (2012)

64. Zhou, H.-J., Parlati, F. \& Wustrow, D. Methods and compositions for JAMM protease inhibition WO2013123071 (A1) (2013).

65. Zhou, H.-J. \& Wustrow, D. Compositions and methods for JAMM protein inhibition. WO2014066506 (A2) (2014).

66. O'Connor, M. J. Targeting the DNA Damage response in cancer. Mol. Cell 60, 547-560 (2015).

67. Jackson, S. P. \& Bartek, J. The DNA-damage response in human biology and disease. Nature $\mathbf{4 6 1}$ 1071-1078 (2009)

68. Jacq, X., Kemp, M., Martin, N. M. \& Jackson, S. P. Deubiquitylating enzymes and DNA damage response pathways. Cell Biochem. Biophys. 67, 25-43 (2013)

69. Castella, M. et al. FANCI Regulates recruitment of the FA core complex at sites of DNA damage independently of FANCD2. PLoS Genet. 11 e1005563 (2015)

70. Nijman, S. M. et al. The Deubiquitinating enzyme USP 1 regulates the Fanconi anemia pathway. Mol. Cell 17, 331-339 (2005)

71. Huang, T. T. et al. Regulation of monoubiquitinated PCNA by DUB autocleavage. Nat. Cell Biol. 8 , 339-347 (2006)

72. Guervilly, J. H., Renaud, E., Takata, M. \& Rosselli, F USP 1 deubiquitinase maintains phosphorylated CHK 1 by limiting its DDB 1 -dependent degradation. Hum. Mol. Genet. 20, 2171-2181 (2011).

73. Williams, S. A. et al. USP1 Deubiquitinates ID proteins to preserve a mesenchymal stem cell program in osteosarcoma. Cell 146, 918-930 (2011).

74. Cohn, M. A. et al. A UAF1-containing multisubunit protein complex regulates the Fanconi anemia pathway. Mol. Cell 28, 786-797 (2007).

75. Chen, J. et al. Selective and cell-active inhibitors of the USP1/ UAF1 deubiquitinase complex reverse cisplatin resistance in non-small cell lung cancer cells. Chem. Biol. 18, 1390-1400 (2011).

76. Ritorto, M. S. et al. Screening of DUB activity and specificity by MALDI-TOF mass spectrometry. Nat. Commun. 5, 4763 (2014). Key publication assessing substrate specificity of 42 DUBs and selectivity profiling of 9 reported DUB inhibitors against a panel of 32 DUBs.

77. Dexheimer, T. S. et al. Synthesis and structure-activity relationship studies of

$\mathrm{N}$-benzyl-2-phenylpyrimidin-4-amine derivatives as potent USP1/UAF1 deubiquitinase inhibitors with anticancer activity against nonsmall cell lung cancer. J. Med. Chem. 57, 8099-8110 (2014).

78. Liang, Q. et al. A selective USP1-UAF1 inhibitor links deubiquitination to DNA damage responses. Nat. Chem. Biol. 10, 298-304 (2014). Identification of the USP1 inhibitor ML323 and demonstration that the compound potentiates cisplatin cytotoxicity in non-small-cell lung cancer and osteosarcoma cells.

79. Schoenfeld, A. R., Apgar, S., Dolios, G., Wang, R. \& Aaronson, S. A. BRCA2 is ubiquitinated in vivo and interacts with USP11, a deubiquitinating enzyme that exhibits prosurvival function in the cellular response to DNA damage. Mol. Cell. Biol. 24, 7444-7455 (2004)

80. Wiltshire, T. D. et al. Sensitivity to poly(ADP-ribose) polymerase (PARP) inhibition identifies ubiquitinspecific peptidase 11 (USP11) as a regulator of DNA double-strand break repair. J. Biol. Chem. 285 , 14565-14571 (2010)

81. Orthwein, A. et al. A mechanism for the suppression f homologous recombination in G1 cells. Nature 528, 422-426 (2015)

82. Burkhart, R. A. et al. Mitoxantrone targets human ubiquitin-specific peptidase 11 (USP11) and is a potent inhibitor of pancreatic cancer cell survival. Mol. Cancer Res. 11, 901-911 (2013).

83. Wijnhoven, P. et al. USP4 Auto-Deubiquitylation Promotes Homologous Recombination. Mol. Cell 60 362-373 (2015)

84. McGarry, E. et al. The deubiquitinase USP9X maintains DNA replication fork stability and DNA damage checkpoint responses by regulating CLASPIN during S-phase. Cancer Res. 76, 2384-2393 (2016).

85. Wolfsperger, F. et al. Deubiquitylating enzyme USP9x regulates radiosensitivity in glioblastoma cells by Mcl-1-dependent and -independent mechanisms. Cell Death Dis. 7, e2039 (2016).
86. Kapuria, V. et al. Deubiquitinase inhibition by smallmolecule WP1130 triggers aggresome formation and tumor cell apoptosis. Cancer Res. 70, 9265-9276 (2010).

87. Kapuria, V. et al. A novel small molecule deubiquitinase inhibitor blocks Jak2 signaling through Jak2 ubiquitination. Cell Signal 23, 2076-2085 (2011).

88. Clague, M. J. et al. Deubiquitylases from genes to organism. Physiol. Rev. 93, 1289-1315 (2013).

89. Prives, C. Signaling to $\mathrm{p} 53$ : breaking the MDM2 p53 circuit. Cell 95, 5-8 (1998).

90. Harris, S. L. \& Levine, A. J. The p53 pathway: positive and negative feedback loops. Oncogene $\mathbf{2 4}$ 2899-2908 (2005).

91. Li, M. et al. Deubiquitination of $\mathrm{p} 53$ by HAUSP is an important pathway for p53 stabilization. Nature $\mathbf{4 1 6}$ 648-653 (2002)

92. Li, M., Brooks, C. L., Kon, N. \& Gu, W. A dynamic role of HAUSP in the p53-Mdm2 pathway. Mol. Cell 13, 879-886 (2004).

93. Cummins, J. M. \& Vogelstein, B. HAUSP is required for p53 destabilization. Cell Cycle 3, 689-692 (2004).

94. van der Horst, A. et al. FOXO4 transcriptional activity is regulated by monoubiquitination and USP7/HAUSP. Nat. Cell Biol. 8, 1064-1073 (2006).

95. Song, M. S. et al. The deubiquitinylation and localization of PTEN are regulated by a HAUSP-PML network. Nature 455, 813-817 (2008)

96. Lecona, E. et al. USP7 is a SUMO deubiquitinase essential for DNA replication. Nat. Struct. Mol. Biol. 23, 270-277 (2016)

97. Colland, F. et al. Small-molecule inhibitor of USP7 HAUSP ubiquitin protease stabilizes and activates p53 in cells. Mol. Cancer Ther. 8, 2286-2295 (2009).

98. Reverdy, C. et al. Discovery of specific inhibitors of human USP7/HAUSP deubiquitinating enzyme. Chem. Biol. 19, 467-477 (2012).

99. Chauhan, D. et al. A small molecule inhibitor of ubiquitin-specific protease-7 induces apoptosis in multiple myeloma cells and overcomes bortezomib resistance. Cancer Cell 22, 345-358 (2012).

100. Fan, Y. H. et al. USP7 inhibitor P22077 inhibits neuroblastoma growth via inducing p53-mediated apoptosis. Cell Death Dis. 4, e867 (2013)

101. Gavory, G. et al. Discovery and characterization of novel, highly potent and selective USP7 inhibitors. Cancer Res. 75 (Suppl. 15), abstr. LB-257 (2015)

102. Liu, H. et al. The Machado-Joseph disease deubiquitinase ataxin-3 regulates the stability and apoptotic function of p53. PLoS Biol. 14, e2000733 (2016).

103. Cuella-Martin, R. et al. 53BP1 integrates DNA repair and $\mathrm{p} 53$-dependent cell fate decisions via distinct mechanisms. Mol. Cell 64, 51-64 (2016).

104. Popov, N. et al. The ubiquitin-specific protease USP28 is required for MYC stability. Nat. Cell Biol. 9 , 765-774 (2007).

105. Diefenbacher, M. E. et al. The deubiquitinase USP28 controls intestinal homeostasis and promotes colorectal cancer. J. Clin. Invest. 124, 3407-3418 (2014).

106. Biju, M. et al. Discovery of potent and selective small molecule USP20 inhibitors. Presented at Ubiquitin Drug Discovery and Diagnostics Conference, Philadelphia, 2012.

107. Mizuno, E. et al. Regulation of epidermal growth factor receptor down-regulation by UBPY-mediated deubiquitination at endosomes. Mol. Biol. Cell 16, 5163-5174 (2005)

108. Niendorf, S. et al. Essential role of ubiquitin-specific protease 8 for receptor tyrosine kinase stability and endocytic trafficking in vivo. Mol. Cell. Biol. 27 5029-5039 (2007).

109. Yewale, C., Baradia, D., Vhora, I., Patil, S. \& Misra, A. Epidermal growth factor receptor targeting in cancer: a review of trends and strategies. Biomaterials 34 8690-8707 (2013)

110. Colombo, M. et al. Synthesis and biological evaluation of 9-oxo-9H-indeno[1,2-b] pyrazine-2,3-dicarbonitrile analogues as potential inhibitors of deubiquitinating enzymes. ChemMedChem 5, 552-558 (2010).

111. Byun, S. et al. USP8 is a novel target for overcoming gefitinib resistance in lung cancer. Clin. Cancer Res. 19, 3894-3904 (2013).

112. Inui, M. et al. USP15 is a deubiquitylating enzyme for receptor-activated SMADs. Nat. Cell Biol. 13 1368-1375 (2011).

113. Wilkinson, K. D. et al. The neuron-specific protein PGP 9.5 is a ubiquitin carboxyl-terminal hydrolase. Science 246, 670-673 (1989). 
114. Day, I. N. \& Thompson, R. J. UCHL1 (PGP 9.5): neuronal biomarker and ubiquitin system protein Progress Neurobiol. 90, 327-362 (2010)

115. Hurst-Kennedy, J., Chin, L. S. \& Li, L. Ubiquitin C-Terminal Hydrolase L1 in Tumorigenesis. Biochem. Res. Int. 2012, 123706 (2012).

116. Hussain, S. et al. The de-ubiquitinase UCH-L1 is an oncogene that drives the development of lymphoma in vivo by deregulating PHLPP 1 and Akt signaling. Leukemia 24, 1641-1655 (2010).

117. Kim, H. J. et al. Ubiquitin C-terminal hydrolase-L1 is a key regulator of tumor cell invasion and metastasis. Oncogene 28, 117-127 (2009).

118. Jang, M. J., Baek, S. H. \& Kim, J. H. UCH-L1 promotes cancer metastasis in prostate cancer cells through EMT induction. Cancer Lett. 302, 128-135 (2011).

119. Gu, Y. Y. et al. The de-ubiquitinase UCHL1 promotes gastric cancer metastasis via the Akt and Erk $1 / 2$ pathways. Tumour Biol. 36, 8379-8387 (2015).

120. Liu, Y. et al. Discovery of inhibitors that elucidate the role of UCH-L1 activity in the $\mathrm{H} 1299$ lung cancer cell line. Chem. Biol. 10, 837-846 (2003).

121. Mermerian, A. H., Case, A., Stein, R. L. \& Cuny, G. D. Structure-activity relationship, kinetic mechanism, and selectivity for a new class of ubiquitin C-terminal hydrolase-L1 (UCH-L1) inhibitors. Bioorg. Med. Chem. Lett. 17, 3729-3732 (2007).

122. Davies, C. W. et al. The co-crystal structure of ubiquitin carboxy-terminal hydrolase L1 (UCHL1) with a tripeptide fluoromethyl ketone (Z-VAE(OMe)FMK). Bioorg. Med. Chem. Lett. 22, 3900-3904 (2012).

123. Jones, A. et al. Novel Compounds. WO2016046530 (2016).

First patent from Mission Therapeutics describing its DUB inhibitors.

124. Kemp, M., Stockley, M. \& Jones, A. Cyanopyrrolidines as DUB inhibitors for the treatment of cancers. WO2017009650 (2017)

125. Hussain, S., Bedekovics, T., Chesi, M., Bergsagel, P. L. $\&$ Galardy, P. J. UCHL1 is a biomarker of aggressive multiple myeloma required for disease progression. Oncotarget 6, 40704-40718 (2015)

126. Schrecengost, R. S. et al. USP22 regulates oncogenic signaling pathways to drive lethal cancer progression. Cancer Res. 74, 272-286 (2014).

127. Liu, Y. L., Yang, Y. M., Xu, H. \& Dong, X. S. Aberrant expression of USP22 is associated with liver metastasis and poor prognosis of colorectal cancer. J. Surg. Oncol. 103, 283-289 (2011).

128. Zhang, Y. et al. Elevated expression of USP22 in correlation with poor prognosis in patients with invasive breast cancer. J. Cancer Res. Clin. Oncol. 137, 1245-1253 (2011)

129. Li, J., Wang, Z. \& Li, Y. USP22 nuclear expression is significantly associated with progression and unfavorable clinical outcome in human esophageal squamous cell carcinoma. J. Cancer Res. Clin. Oncol. 138, 1291-1297 (2012).

130. Piao, S et al. USP22 is useful as a novel molecular marker for predicting disease progression and patient prognosis of oral squamous cell carcinoma. PLOS ONE 7, e42540 (2012).

131. Zhang, J. et al. Identification of the E3 deubiquitinase ubiquitin-specific peptidase 21 (USP21) as a positive regulator of the transcription factor GATA3. J. Biol. Chem. 288, 9373-9382 (2013). This publication demonstrates that USP2 1 deubiquitylates and stabilizes the transcription factor GATA3 in $\mathrm{T}_{\text {reg }}$ cells.

132. Li, Y. et al. USP21 prevents the generation of T-helper-1-like Treg cells. Nat. Commun. 7, 13559 (2016).

133. Facciabene, A., Motz, G. T. \& Coukos, G. T-Regulatory cells: key players in tumor immune escape and angiogenesis. Cancer Res. 72, 2162-2171 (2012).

134. Ross, C. A. \& Pickart, C. M. The ubiquitin-proteasome pathway in Parkinson's disease and other neurodegenerative diseases. Trends Cell Biol. 14, 703-711 (2004)

135. Todi, S. V. \& Paulson, H. L. Balancing act: deubiquitinating enzymes in the nervous system. Trends Neurosci. 34, 370-382 (2011).

136. Ristic, G., Tsou, W. L. \& Todi, S. V. An optimal ubiquitin-proteasome pathway in the nervous system the role of deubiquitinating enzymes. Frontiers $\mathrm{Mol}$. Neurosci. 7, 72 (2014).

137. Green, D. R., Galluzzi, L. \& Kroemer, G Mitochondria and the autophagy-inflammation-cell death axis in organismal aging. Science 333, 1109-1112 (2011).
138. Ross, J. M., OIson, L. \& Coppotelli, G. Mitochondrial and ubiquitin proteasome system dysfunction in ageing and disease: two sides of the same coin? Int. J. Mol. Sci. 16, 19458-19476 (2015).

139. Martin, I., Dawson, V. L. \& Dawson, T. M. Recent advances in the genetics of Parkinson's disease. Annu. Rev. Genom. Hum. Genet. 12, 301-325 (2011).

140. Hauser, D. N. \& Hastings, T. G. Mitochondrial dysfunction and oxidative stress in Parkinson's disease and monogenic parkinsonism. Neurobiol. Dis. $\mathbf{5 1}$ 35-42 (2013).

141. Narendra, D. P. \& Youle, R. J. Targeting mitochondrial dysfunction: role for PINK1 and Parkin in mitochondrial quality control. Antioxid. Redox Signal. 14, 1929-1938 (2011).

142. Bingol, B. et al. The mitochondrial deubiquitinase USP30 opposes parkin-mediated mitophagy. Nature 510, 370-375 (2014)

This key publication demonstrates that USP30 antagonizes mitophagy driven by the ubiquitin ligase parkin and protein kinase PINK1.

143. Durcan, T. M. \& Fon, E. A. The three 'P's of mitophagy: PARKIN, PINK1, and post-translational modifications. Genes Dev. 29, 989-999 (2015).

144. Nakamura, N. $\&$ Hirose, S. Regulation of mitochondrial morphology by USP30, a deubiquitinating enzyme present in the mitochondrial outer membrane. Mol. Biol. Cell 19, 1903-1911 (2008).

145. Narendra, D., Tanaka, A., Suen, D. F. \& Youle, R. J. Parkin is recruited selectively to impaired mitochondria and promotes their autophagy. J. Cell Biol. 183, 795-803 (2008).

146. Yue, W. et al. A small natural molecule promotes mitochondrial fusion through inhibition of the deubiquitinase USP30. Cell Res. 24, 482-496 (2014).

147. Jones, A., Kemp, M., Stockley, M., Gibson, K. R. \& Whitlock, G. A. Cyano-pyrrolidine compounds as USP30 inhibitors. WO2016156816 (2016)

148. Durcan, T. M. et al. USP8 regulates mitophagy by removing K6-linked ubiquitin conjugates from parkin. EMBO J. 33, 2473-2491 (2014)

149. Alexopoulou, Z. et al. Deubiquitinase Usp8 regulates alpha-synuclein clearance and modifies its toxicity in Lewy body disease. Proc. Natl Acad. Sci. USA 113 E4688-E4697 (2016)

150. Cornelissen, T. et al. The deubiquitinase USP 15 antagonizes Parkin-mediated mitochondrial ubiquitination and mitophagy. Hum. Mol. Genet. 23 5227-5242 (2014)

151. Durcan, T. M., Kontogiannea, M., Bedard, N., Wing, S. S. \& Fon, E. A. Ataxin-3 deubiquitination is coupled to Parkin ubiquitination via E2 ubiquitinconjugating enzyme. J. Biol. Chem. 287, 531-541 (2012)

152. Guterman, A. \& Glickman, M. H. Deubiquitinating enzymes are IN/(trinsic to proteasome function). Curr. Protein Peptide Sci. 5, 201-211 (2004).

153. Finley, D., Gahman, T. C., King, R. W., Lee, B. H. \& Lee, M. J. Tricyclic proteasome activity enhancing compounds. WO 2012012712 (2012).

154. Chambers, R. J., Foley, M. \& Tait, B. Proteasome activity modulating compounds. WO 201311265 (2013)

155. Chambers, R. J., Foley, M. \& Tait, B. Proteasome activity enhancing compounds. WO2013112699 (2013)

References 154 and 155 are Proteostasis Therapeutics patents that describe USP14 inhibitors.

156. Crimmins, S. et al. Transgenic rescue of ataxia mice with neuronal-specific expression of ubiquitinspecific protease 14. J. Neurosci. 26 11423-11431 (2006)

157. Ortuno, D., Carlisle, H. J. \& Miller, S. Does inactivation of USP 14 enhance degradation of proteasomal substrates that are associated with neurodegenerative diseases? F1000Res. 5, 137 (2016).

158. Joo, H. Y. et al. Regulation of cell cycle progression and gene expression by $\mathrm{H} 2 \mathrm{~A}$ deubiquitination. Nature 449, 1068-1072 (2007)

159. Adorno, M. et al. Usp 16 contributes to somatic stemcell defects in Down's syndrome. Nature 501 380-384 (2013).

This work demonstrates that USP16 has an important role in antagonizing the self-renewal and/or senescence pathways in Down syndrome.

160. Xu, J. C., Dawson, V. L. \& Dawson, T. M. Usp 16: key controller of stem cells in Down syndrome. $E M B O \mathrm{~J}$. 32, 2788-2789 (2013)
161. Hu, H \& Sun, S. C. Ubiquitin signaling in immune responses. Cell Res. 26, 457-483 (2016). A recent review that describes how ubiquitylation regulates immune signalling and discusses of roles of many DUBs.

162. Jiang, X. \& Chen, Z. J. The role of ubiquitylation in immune defence and pathogen evasion. Nat. Rev. Immunology 12, 35-48 (2011).

163. Adhikari, A., Xu, M. \& Chen, Z. J. Ubiquitin-mediated activation of TAK 1 and IKK. Oncogene 26 3214-3226 (2007).

164. Tokunaga, F. Linear ubiquitination-mediated NF-kappaB regulation and its related disorders J. Biochem. 154, 313-323 (2013).

165. Harhaj, E. W. \& Dixit, V. M. Deubiquitinases in the regulation of NF-kappaB signaling. Cell Res. 21, 22-39 (2011).

166. Catrysse, L., Vereecke, L., Beyaert, R. \& van Loo, G A20 in inflammation and autoimmunity. Trends Immunol. 35, 22-31 (2014).

167. Wertz, I. E. et al. De-ubiquitination and ubiquitin ligase domains of A20 downregulate NF-kappaB signalling. Nature 430, 694-699 (2004). This publication demonstrates that phosphorylation of A20 promotes cleavage of K63-linked polyubiquitin chains.

168. Skaug, B. et al. Direct, noncatalytic mechanism of IKK inhibition by A20. Mol. Cell 44, 559-571 (2011).

169. Wertz, I. E. et al. Phosphorylation and linear ubiquitin direct A20 inhibition of inflammation. Nature $\mathbf{5 2 8}$ 370-375 (2015)

170. Zhang, M. et al. Regulation of IkappaB kinaserelated kinases and antiviral responses by tumor suppressor CYLD. J. Biol. Chem. 283 18621-18626 (2008)

171. Friedman, C. S. et al. The tumour suppressor CYLD is a negative regulator of RIG-I-mediated antiviral response. EMBO Rep. 9, 930-936 (2008).

172. Fiil, B. K. \& Gyrd-Hansen, M. Met1-linked ubiquitination in immune signalling. FEBS J. 281 4337-4350 (2014).

173. Iwai, K., Fujita, H. \& Sasaki, Y. Linear ubiquitin chains: NF-kappaB signalling, cell death and beyond. Nat. Rev. Mol. Cell Biol. 15, 503-508 (2014).

174. MacDuff, D. A. et al. Phenotypic complementation of genetic immunodeficiency by chronic herpesvirus infection. eLife https://dx.doi.org/10.7554/ eLife.04494.002 (2015)

175. Gerlach, B. et al. Linear ubiquitination prevents inflammation and regulates immune signalling. Nature 471, 591-596 (2011).

176. Ikeda, F. et al. SHARPIN forms a linear ubiquitin ligase complex regulating NF-kappaB activity and apoptosis. Nature 471, 637-641 (2011)

177. Tokunaga, F. et al. SHARPIN is a component of the NF-kappaB-activating linear ubiquitin chain assembly complex. Nature 471, 633-636 (2011).

178. Tokunaga, F. et al. Involvement of linear polyubiquitylation of NEMO in NF-kappaB activation Nat. Cell Biol. 11, 123-132 (2009).

179. Boisson, B. et al. Human HOIP and LUBAC deficiency underlies autoinflammation, immunodeficiency, amylopectinosis, and lymphangiectasia. J. Exp. Med. 212, 939-951 (2015).

180. Boisson, B. et al. Immunodeficiency, autoinflammation and amylopectinosis in humans with inherited HOIL-1 and LUBAC deficiency. Nature Immunol. 13, 1178-1186 (2012)

181. Keusekotten, K. et al. OTULIN Antagonizes LUBAC Signaling by Specifically Hydrolyzing Met 1 -Linked Polyubiquitin. Cell 153, 1312-1326 (2013)

182. Rivkin, E. et al. The linear ubiquitin-specific deubiquitinase gumby regulates angiogenesis. Nature 498, 318-324 (2013).

Together with reference 181 , this is a key publication that identifies OTULIN as the DUB that removes linear ubiquitin chains.

183. Damgaard, R. B. et al. The Deubiquitinase OTULIN is an essential negative regulator of inflammation and autoimmunity. Cell 166, 1215-1230.e1220 (2016).

184. Honke, N., Shaabani, N., Zhang, D. E., Hardt, C. \& Lang, K. S. Multiple functions of USP18. Cell Death Dis. 7, e2444 (2016).

185. Rioux, J. D. \& Abbas, A. K. Paths to understanding the genetic basis of autoimmune disease. Nature 435 584-589 (2005)

186. Walsh, K. P. \& Mills, K. H. Dendritic cells and other innate determinants of $\mathrm{T}$ helper cell polarisation. Trends Immunol. 34, 521-530 (2013). 
187. Bettelli, E., Oukka, M. \& Kuchroo, V. K. T -17 cells in the circle of immunity and autoimmunity. Nature Immunol. 8, 345-350 (2007)

188. Bettelli, E., Korn, T., Oukka, M. \& Kuchroo, V. K. Induction and effector functions of $\mathrm{T}_{\mathrm{H}} 17$ cells. Nature 453, 1051-1057 (2008)

189. Yang, J. et al. Cutting edge: Ubiquitin-specific protease 4 promotes Th 17 cell function under inflammation by deubiquitinating and stabilizing RORgammat. J. Immunol. 194, 4094-4097 (2015).

190. Okada, K. et al. Vialinin A is a ubiquitin-specific peptidase inhibitor. Bioorg. Med. Chem. Lett. 23 4328-4331 (2013).

191. Jin, J. et al. Epigenetic regulation of the expression of II 12 and II 23 and autoimmune inflammation by the deubiquitinase Trabid. Nature Immunol. 17, 259-268 (2016).

192. Liu, X. et al. USP18 inhibits NF-kappaB and NFAT activation during Th17 differentiation by deubiquitinating the TAK1-TAB1 complex. J. Exp. Med. 210, 1575-1590 (2013).

193. Hu, H. et al. Otud7b facilitates T cell activation and inflammatory responses by regulating Zap70 ubiquitination. J. Exp. Med. 213, 399-414 (2016).

194. Wang, H. et al. ZAP-70: an essential kinase in T-cell signaling. Cold Spring Harb. Perspect. Biol. 2, a002279 (2010)

195. Damsker, J. M., Hansen, A. M. \& Caspi, R. R. Th1 and Th17 cells: adversaries and collaborators. Ann. NY Acad. Sci. 1183, 211-221 (2010).

196. Pan, L. et al. Deubiquitination and stabilization of T-bet by USP10. Biochem. Biophys. Res. Commun. 449, 289-294 (2014).

197. Bailey-Elkin, B. A., van Kasteren, P. B., Snijder, E. J. Kikkert, M. \& Mark, B. L. Viral OTU deubiquitinases: a structural and functional comparison. PLoS Pathog. 10, e 1003894 (2014).

198. Sun, L et al Coronavirus papain-like proteases negatively regulate antiviral innate immune response through disruption of STING-mediated signaling. PLOS ONE 7, e30802 (2012)

199. Ratia, K. et al. A noncovalent class of papain-like protease/deubiquitinase inhibitors blocks SARS virus replication. Proc. Natl Acad. Sci. USA 105 16119-16124 (2008)

200. Bailey-Elkin, B. A. et al. Crystal structure of the Middle East respiratory syndrome coronavirus (MERS-CoV) papain-like protease bound to ubiquitin facilitates targeted disruption of deubiquitinating activity to demonstrate its role in innate immune suppression. J. Biol. Chem. 289, 34667-34682 (2014).

201. Lei, J et al. Crystal structure of the papain-like protease of MERS coronavirus reveals unusual, potentially druggable active-site features. Antiviral Res. 109, 72-82 (2014).

202. Frias-Staheli, N. et al. Ovarian tumor domaincontaining viral proteases evade ubiquitin- and ISG15-dependent innate immune responses. Cell Host Microbe 2, 404-416 (2007).

203. Pruneda, J. N. et al. The molecular basis for ubiquitin and ubiquitin-like specificities in bacterial effector proteases. Mol. Cell 63 261-276 (2016)

204. Artavanis-Tsakonas, K. et al. Identification by functional proteomics of a deubiquitinating/ deNeddylating enzyme in Plasmodium falciparum. Mol. Microbiol 61, 1187-1195 (2006).

205. Frickel, E. M. et al. Apicomplexan UCHL3 retains dual specificity for ubiquitin and Nedd8 throughout evolution. Cell. Microbiol. 9, 1601-1610 (2007).

206. Artavanis-Tsakonas, K. et al. Characterization and structural studies of the Plasmodium falciparum ubiquitin and Nedd8 hydrolase UCHL3. J. Biol. Chem. 285, 6857-6866 (2010)

207. Wrigley, J. D. et al. Enzymatic characterisation of USP7 deubiquitinating activity and inhibition Cell Biochem. Biophys. 60, 99-111 (2011).

208. Sahtoe, D. D. \& Sixma, T. K. Layers of DUB regulation Trends Biochem. Sci. 40, 456-467 (2015). This review discusses the extent and variety of allosteric regulation modes of DUB catalytic activity.

209. Mevissen, T. E. T. \& Komander, D. Mechanisms of deubiquitinase specificity and regulation. Annu. Rev. Biochem. 86, 159-192 (2017). A very recent and comprehensive review that describes DUB substrate specificity and regulation of catalytic activity.
210. Kemp, M. Recent advances in the discovery of deubiquitinating enzyme inhibitors. Progress Med. Chem. 55, 149-192 (2016).

211. Kathman, S. G., Xu, Z. \& Statsyuk, A. V. A fragmentbased method to discover irreversible covalent inhibitors of cysteine proteases. J. Med. Chem. 57, 4969-4974 (2014).

212. Donato, N. J. et al. Deubiquitinase inhibitors and methods for use of the same. WO2015054555 (2015)

213. Turk, B. Targeting proteases: successes, failures and future prospects. Nat. Rev. Drug Discov. 5, 785-799 (2006)

214. Hershko, A., Ciechanover, A., Heller, H., Haas, A. L. \& Rose, I. A. Proposed role of ATP in protein breakdown: conjugation of protein with multiple chains of the polypeptide of ATP-dependent proteolysis. Proc. Natl Acad. Sci. USA 77, 1783-1786 (1980).

215. Nijman, S. M. et al. A genomic and functional inventory of deubiquitinating enzymes. Cell 123 , 773-786 (2005)

216. Faesen, A. C. et al. Mechanism of USP7/HAUSP activation by its $\mathrm{C}$-terminal ubiquitin-like domain and allosteric regulation by GMP-synthetase. Mol. Cell 44, 147-159 (2011).

217. Finley, D. Recognition and processing of ubiquitin protein conjugates by the proteasome. Annu. Rev. Biochem. 78, 477-513 (2009).

218. Meyer, H. \& Weihl, C. C. The VCP/p97 system at a glance: connecting cellular function to disease pathogenesis. J. Cell Sci. 127, 3877-3883 (2014).

219. Kato, J. Y. \& Yoneda-Kato, N. Mammalian COP9 signalosome. Genes Cells 14, 1209-1225 (2009)

220. Sahtoe, D. D., van Dijk, W. J., Ekkebus, R., Ovaa, H. \& Sixma, T. K. BAP1/ASXL1 recruitment and activation for H2A deubiquitination. Nat. Commun. 7, 10292 (2016).

221. Ventii, K. H. \& Wilkinson, K. D. Protein partners of deubiquitinating enzymes. Biochem. J. 414, 161-175 (2008)

222. D'Andrea, A. Inhibitors of USP 1 deubiquitinating enzyme complex. US2008167229 (2008).

223. Guedat, P. et al. Novel tetracyclic inhibitors of cysteine proteases, the pharmaceutical compositions thereof and their therapeutic applications. US2008103149 (2008)

224. Colland, F \& Gourdel, M. E. Selective and reversible inhibitors of ubiquitin specific protease 7 . WO2013030218 (2013)

225. Dang, L. C., Melandri, F. D. \& Stein, R. L. Kinetic and mechanistic studies on the hydrolysis of ubiquitin C-terminal 7-amido-4-methylcoumarin by deubiquitinating enzymes. Biochemistry 37 1868-1879 (1998)

226. de Jong, A. et al. Ubiquitin-based probes prepared by total synthesis to profile the activity of deubiquitinating enzymes. Chembiochem 13 2251-2258 (2012)

227. McGouran, J. F. et al. Fluorescence-based active site probes for profiling deubiquitinating enzymes. Org. Biomol. Chem. 10, 3379-3383 (2012).

228. Orcutt, S. J., Wu, J., Eddins, M. J., Leach, C. A \& Strickler, J. E. Bioluminescence assay platform for selective and sensitive detection of Ub/Ubl proteases. Biochim. Biophys. Acta 1823, 2079-2086 (2012).

229. Leach, C. A., Strickler, J. E. \& Eddins, M. J. Methods of screening for inhibitors of enzymes. WO2013043970 (2013)

230. Komander, D. \& Rape, M. The ubiquitin code. Annu. Rev. Biochem. 81, 203-229 (2012). A landmark review of the broad scope of ubiquitin modifications, illustrating the large diversity of substrates handled by DUBs.

231. Hospenthal, M. K., Mevissen, T. E. \& Komander, D. Deubiquitinase-based analysis of ubiquitin chain architecture using Ubiquitin Chain Restriction (UbiCRest). Nat. Protoc. 10, 349-361 (2015)

232. Sowa, M. E., Bennett, E. J., Gygi, S. P. \& Harper, J. W. Defining the human deubiquitinating enzyme interaction landscape. Cell 138, 389-403 (2009) This work describes the extensive landscape of protein-protein interactions engaged by DUBs.

233. Harrigan, J. \& Jacq, X. Monitoring Target Engagement of Deubiquitylating Enzymes Using Activity Probes: Past, Present, and Future. Methods Mol. Biol. 1449, 395-410 (2016).

A recent review of the utility and benefits of activity probes to monitor DUB target engagement in cells and tissues.

234. Hewings, D. S., Flygare, J. A., Bogyo, M. \& Wertz, I. E. Activity-based probes for the ubiquitin conjugationdeconjugation machinery: new chemistries, new tools, and new insights. FEBS J. 284, 1555-1576 (2017).
235. Borodovsky, A. et al. A novel active site-directed probe specific for deubiquitylating enzymes reveals proteasome association of USP 14. EMBO J. 20, 5187-5196 (2001)

236. Galardy, P., Ploegh, H. L. \& Ovaa, H. Mechanismbased proteomics tools based on ubiquitin and ubiquitin-like proteins: crystallography, activity profiling, and protease identification. Methods Enzymol. 399, 120-131 (2005)

237. El Oualid, F. et al. Chemical synthesis of ubiquitin ubiquitin-based probes, and diubiquitin. Angew. Chem. Int. Ed. 49, 10149-10153 (2010).

238. Kim, W. et al. Systematic and quantitative assessment of the ubiquitin-modified proteome. Mol. Cell 44, 325-340 (2011)

239. Altun, M. et al. Activity-based chemical proteomics accelerates inhibitor development for deubiquitylating enzymes. Chem. Biol. 18, 1401-1412 (2011). Detailed analysis of DUB activity using ubiquitin-based activity probes and characterization of the selectivity of the DUB inhibitors PR-619 and P22077.

240. Wang, X. et al. The 19S Deubiquitinase inhibitor b-AP15 is enriched in cells and elicits rapid commitment to cell death. Mol. Pharmacol. 85, 932-945 (2014).

241. Chen, Y. et al. Expression and clinical significance of $\mathrm{UCH} 37$ in human esophageal squamous cell carcinoma. Dig. Dis. Sci. 57, 2310-2317 (2012).

242. Gray, D. A. et al. Elevated expression of Unph, a protooncogene at 3p21.3, in human lung tumors. Oncogene 10, 2179-2183 (1995).

243. Zhang, L. et al. USP4 is regulated by AKT phosphorylation and directly deubiquitylates TGF-beta type I receptor. Nat. Cell Biol. 14, 717-726 (2012).

244. Heo, M. J. et al. microRNA-148a dysregulation discriminates poor prognosis of hepatocellular carcinoma in association with USP4 overexpression. Oncotarget 5, 2792-2806 (2014).

245. Bayraktar, S. et al. USP-11 as a predictive and prognostic factor following neoadjuvant therapy in women with breast cancer. Cancer J. 19, 10-17 (2013).

246. Huether, R. et al. The landscape of somatic mutations in epigenetic regulators across 1,000 paediatric cancer genomes. Nat. Commun. 5, 3630 (2014).

247. Ma, M. \& Yu, N. Ubiquitin-specific protease expression is a prognostic factor in epithelial ovarian cancer and correlates with lymph node metastasis. Onco Targets Ther. 9, 1559-1569 (2016).

248. Masuya, D. et al. The HAUSP gene plays an important role in non-small cell lung carcinogenesis through p53-dependent pathways. J. Pathol. 208, 724-732 (2006).

249. Zhao, G. Y. et al. USP7 overexpression predicts a poor prognosis in lung squamous cell carcinoma and large cell carcinoma. Tumour Biol. 36, 1721-1729 (2015).

250. Forbes, S. A. et al. COSMIC: somatic cancer genetics at high-resolution. Nucleic Acids Res. 45, D777-D783 (2017).

251. Mermel, C. H. et al. GISTIC2.0 facilitates sensitive and confident localization of the targets of focal somatic copy-number alteration in human cancers. Genome Biol. 12, R41 (2011).

252. Zheng, S et al. Heterogeneous expression and biological function of ubiquitin carboxy-terminal hydrolase-L1 in osteosarcoma. Cancer Lett. 359 36-46 (2015).

253. Akishima-Fukasawa, Y. et al. Significance of PGP9.5 expression in cancer-associated fibroblasts for prognosis of colorectal carcinoma. Am. J. Clin. Pathol. 134, 71-79 (2010)

254. Ma, Y. et al. Proteomic profiling of proteins associated with lymph node metastasis in colorectal cancer. J. Cell. Biochem. 110, 1512-1519 (2010).

255. Goto, Y. et al. UCHL1 provides diagnostic and antimetastatic strategies due to its deubiquitinating effect on HIF-1 alpha. Nat. Commun. 6, 6153 (2015).

256. Gunia, S. et al. Protein gene product 9.5 is diagnostically helpful in delineating high-grade renal cell cancer involving the renal medullary/sinus region from invasive urothelial cell carcinoma of the renal pelvis. Hum. Pathol. 44, 712-717 (2013).

257. Otsuki, T. et al. Expression of protein gene product 9.5 (PGP9.5)/ubiquitin-C-terminal hydrolase 1 (UCHL-1) in human myeloma cells. Br. J. Haematol. 127, 292-298 (2004).

258. Hibi, K. et al. PGP9.5 as a candidate tumor marke for non-small-cell lung cancer. Am. J. Pathol. 155 , 711-715 (1999). 
259. Hu, J. et al. Expression patterns of USP22 and potential targets BMI-1, PTEN, p-AKT in non-smallcell lung cancer. Lung cancer 77, 593-599 (2012).

260. Liang, J. X. et al. Ubiquitin specific protease 22 -induced autophagy is correlated with poor prognosis of pancreatic cancer. Oncol. Rep. 32 2726-2734 (2014).

261. Cunningham, C. N. et al. USP30 and parkin homeostatically regulate atypical ubiquitin chains on mitochondria. Nat. Cell Biol. 17, 160-169 (2015).

262. Wilson, S. M. et al. Synaptic defects in ataxia mice result from a mutation in Usp 14, encoding a ubiquitinspecific protease. Nat. Genet. 32, 420-425 (2002).

263. Ceriani, M., Amigoni, L., D’Aloia, A., Berruti, G. \& Martegani, E. The deubiquitinating enzyme UBPy/ USP8 interacts with TrkA and inhibits neuronal differentiation in PC1 2 cells. Exp. Cell Res. 333 , 49-59 (2015)

264. Daviet, L. \& Colland, F. Targeting ubiquitin specific proteases for drug discovery. Biochimie 90, 270-283 (2008).

265. Bruzzone, F., Vallarino, M., Berruti, G. \& Angelini, C. Expression of the deubiquitinating enzyme mUBPy in the mouse brain. Brain Res. 1195, 56-66 (2008).

266. Yang, W. et al. The histone $\mathrm{H} 2 \mathrm{~A}$ deubiquitinase Usp 16 regulates embryonic stem cell gene expression and lineage commitment. Nat. Commun. 5, 3818 (2014).

267. Ovaa, H. et al. Activity-based ubiquitin-specific protease (USP) profiling of virus-infected and malignant human cells. Proc. Natl Acad. Sci. USA 101 2253-2258 (2004).

268. Liu, L. Q. et al. A novel ubiquitin-specific protease, UBP43, cloned from leukemia fusion protein AML1-ETO-expressing mice, functions in hematopoietic cell differentiation. Mol. Cell. Biol. 19 3029-3038 (1999).

269. Malakhova, O. A. et al. UBP43 is a novel regulator of interferon signaling independent of its ISG 15 isopeptidase activity. EMBO J. 25, 2358-2367 (2006).

270. Zhong, B. et al. Ubiquitin-specific protease 25 regulates TLR4-dependent innate immune responses through deubiquitination of the adaptor protein TRAF3. Sci. Signal 6, ra35 (2013).

271. Lin, D. et al. Induction of USP25 by viral infection promotes innate antiviral responses by mediating the stabilization of TRAF3 and TRAF6. Proc. Natl Acad. Sci. USA 112, 11324-11329 (2015)

272. Ren, Y. et al. The Type I Interferon-IRF7 Axis Mediates Transcriptional Expression of Usp25 Gene. J. Biol. Chem. 291, 13206-13215 (2016).

273. Liu, Y. C., Penninger, J. \& Karin, M. Immunity by ubiquitylation: a reversible process of modification. Nat. Rev. Immunology 5, 941-952 (2005).

274. Wertz, I. \& Dixit, V. A20-a bipartite ubiquitin editing enzyme with immunoregulatory potential. Adv. Exp. Med. Biol. 809, 1-12 (2014).

275. Wang, L. et al. USP4 positively regulates RIG-I-mediated antiviral response through deubiquitination and stabilization of RIG-I. J. Virol. 87, 4507-4515 (2013).

276. Fan, Y. H. et al. USP4 targets TAK1 to downregulate TNFalpha-induced NF-kappaB activation. Cell Death Differ. 18, 1547-1560 (2011)
277. Han, L et al. The E3 deubiquitinase USP 17 is a positive regulator of retinoic acid-related orphan nuclear receptor gammat (RORgammat) in Th 17 cells. J. Biol. Chem. 289, 25546-25555 (2014).

278. Ni, Y. et al. The deubiquitinase USP17 regulates the stability and nuclear function of IL-33. Int. J. Mol. SCi. 16, 27956-27966 (2015)

279. Lim, K. H., Ramakrishna, S. \& Baek, K. H. Molecular mechanisms and functions of cytokine-inducible deubiquitinating enzymes. Cytokine Growth Factor Rev. 24, 427-431 (2013).

280. Annunziata, C. M. et al. Frequent engagement of the classical and alternative NF-kappaB pathways by diverse genetic abnormalities in multiple myeloma. Cancer Cell 12, 115-130 (2007).

281. Keats, J. J. et al. Promiscuous mutations activate the noncanonical NF-kappaB pathway in multiple myeloma. Cancer Cell 12, 131-144 (2007).

282. Hellerbrand, C. et al. Reduced expression of CYLD in human colon and hepatocellular carcinomas. Carcinogenesis 28, 21-27 (2007).

283. Massoumi, R. et al. Down-regulation of CYLD expression by Snail promotes tumor progression in malignant melanoma. J. Exp. Med. 206, 221-232 (2009)

284. Ye, Y. et al. TRE17/USP6 oncogene translocated in aneurysmal bone cyst induces matrix metalloproteinase production via activation of NF-kappaB. Oncogene 29, 3619-3629 (2010).

285. Alwan, H. A. \& van Leeuwen, J. E. UBPY-mediated epidermal growth factor receptor (EGFR) de-ubiquitination promotes EGFR degradation. J. Biol. Chem. 282, 1658-1669 (2007).

286. Harbour, J. W. et al. Frequent mutation of BAP1 in metastasizing uveal melanomas. Science 330 $1410-1413$ (2010)

287. O'Shea, S. J. et al. A population-based analysis of germline BAP1 mutations in melanoma. Hum. Mol. Genet. 26, 717-728 (2017)

288. Testa, J. R. et al. Germline BAP1 mutations predispose to malignant mesothelioma. Nat. Genet. 43, 1022-1025 (2011).

289. Popova, T. et al. Germline BAP1 mutations predispose to renal cell carcinomas. Am. J. Hum. Genet. 92 974-980 (2013).

290. Li, Z., Wang, D., Messing, E. M. \& Wu, G. VHL proteininteracting deubiquitinating enzyme 2 deubiquitinates and stabilizes HIF-1 alpha. EMBO Rep. 6, 373-378 (2005)

291. Malakhov, M. P., Malakhova, O. A., Kim, K. I. Ritchie, K. J. \& Zhang, D. E. UBP43 (USP18) specifically removes ISG 15 from conjugated proteins. J. Biol. Chem. 277, 9976-9981 (2002).

292. Zhong, H. et al. Ubiquitin-specific proteases 25 negatively regulates virus-induced type I interferon signaling. PLOS ONE 8, e80976 (2013).

293. Colleran, A. et al. Deubiquitination of NF-kappaB by ubiquitin-specific protease-7 promotes transcription. Proc. Natl Acad. Sci. USA 110, 618-623 (2013)

294. Zhou, F. et al. Ubiquitin-specific protease 4 mitigates Toll-like/interleukin-1 receptor signaling and regulates innate immune activation. J. Biol. Chem. 287. 11002-11010 (2012)
295. Chen, R et al. The ubiquitin-specific protease 17 is involved in virus-triggered type I IFN signaling. Cell Res. 20, 802-811 (2010).

296. Wang, X. et al. The proteasome deubiquitinase inhibitor VLX1570 shows selectivity for ubiquitinspecific protease-14 and induces apoptosis of multiple myeloma cells. Scientif. Rep. 6, 26979 (2016).

297. Maloney, D. J. et al. Inhibitors of the USP1/UAF1 deubiquitinase complex and uses thereof. WO2014105952 (A2) (2014)

298. Weinstock, J. et al. Selective dual inhibitors of the cancer-related deubiquitylating proteases USP7 and USP47. Acs Med. Chem. Lett. 3, 789-792 (2012).

299. Foley, M., Tait, B. \& Cullen, M. Proteostasis regulators. WO2012154967 (A1) (2012).

300. Finley, D., King, R. W., Lee, B. H., Lee, M. J. \& Gahman, T. C. Compositions and methods for enhancing proteasome activity. WO2011094545 (A2) (2011).

301. Davis, M. I. et al. Small Molecule inhibition of the ubiquitin-specific protease USP2 accelerates cyclin D1 degradation and leads to cell cycle arrest in colorectal cancer and mantle cell lymphoma models. J. Biol. Chem. 291 24628-24640 (2016)

302. Liu, J. et al. Beclin 1 controls the levels of p53 by regulating the deubiquitination activity of USP 10 and USP13. Cell 147, 223-234 (2011).

303. Zhong, B. et al. Negative regulation of IL-17-mediated signaling and inflammation by the ubiquitin-specific protease USP25. Nature Immunol. 13, 1110-1117 (2012)

\section{Acknowledgements}

The authors thank K. Dry for extensive editing and expert advice, and other members of the S.P.J. academic laboratory for helpful discussions and advice. The authors thank L. Greger for the generation of the phylogenic tree. Research in the S.P.J. laboratory is funded by the Cancer Research UK (CRUK) Program Grant C6/A18796 and a Wellcome Trust Investigator Award (206388/Z/17/Z), and institute core infrastructure funding is provided by the CRUK (C6946/A24843) and the Wellcome Trust (WT203144).

\section{Competing interests statement}

The authors declare competing interests: see Web version for details.

\section{Publisher's note}

Springer Nature remains neutral with regard to jurisdictional claims in published maps and institutional affiliations.

FURTHER INFORMATION

Mission Therapeutics pipeline: http://missiontherapeutics. com/pipeline/

Proteostasis pipeline: http://www.proteostasis.com/productpipeline/novel-strategies/

ALL LINKS ARE ACTIVE IN THE ONLINE PDF 